\title{
Neuroligin 2 Is Required for Synapse Development and Function at the Drosophila Neuromuscular Junction
}

\author{
Mingkuan Sun, $, 1,2$ Guanglin Xing, ${ }^{1,2}$ Liudi Yuan, ${ }^{1,3}$ Guangming Gan, ${ }^{1,2}$ David Knight, ${ }^{4}$ Sheila Irene With, ${ }^{4}$ Cui He, ${ }^{1,2}$ \\ Junhai Han, ${ }^{1,2}$ Xiankun Zeng, ${ }^{2}$ Ming Fang, ${ }^{1,2}$ Gabrielle L. Boulianne, ${ }^{4}$ and Wei Xie ${ }^{1,2}$ \\ ${ }^{1}$ The Key Laboratory of Developmental Genes and Human Disease, Ministry of Education, Institute of Life Science, Southeast University, Nanjing 210009, \\ China, Departments of ${ }^{2}$ Medical Genetics and Developmental Biology and ${ }^{3}$ Biochemistry and Molecular Biology, Medical School of Southeast University, \\ Nanjing 210009, China, and ${ }^{4}$ Program in Developmental and Stem Cell Biology, The Hospital for Sick Children and Department Molecular Genetics, \\ University of Toronto, Toronto, Ontario, Canada, M5G 1L7
}

Neuroligins belong to a highly conserved family of cell adhesion molecules that have been implicated in synapse formation and function. However, the precise in vivo roles of Neuroligins remain unclear. In the present study, we have analyzed the function of Drosophila neuroligin $2(d n l 2)$ in synaptic development and function. We show that $d n l 2$ is strongly expressed in the embryonic and larval CNS and at the larval neuromuscular junction (NMJ). dnl2 null mutants are viable but display numerous structural defects at the NMJ, including reduced axonal branching and fewer synaptic boutons. $d n l 2$ mutants also show an increase in the number of active zones per bouton but a decrease in the thickness of the subsynaptic reticulum and length of postsynaptic densities. $d n l 2$ mutants also exhibit a decrease in the total glutamate receptor density and a shift in the subunit composition of glutamate receptors in favor of GluRIIA complexes. In addition to the observed defects in synaptic morphology, we also find that $d n l 2$ mutants show increased transmitter release and altered kinetics of stimulus-evoked transmitter release. Importantly, the defects in presynaptic structure, receptor density, and synaptic transmission can be rescued by postsynaptic expression of $d n l 2$. Finally, we show that $d n l 2$ colocalizes and binds to Drosophila neurexin (dnrx) in vivo. However, whereas homozygous mutants for either $d n l 2$ or $d n r x$ are viable, double mutants are lethal and display more severe defects in synaptic morphology. Altogether, our data show that, although $d n l 2$ is not absolutely required for synaptogenesis, it is required postsynaptically for synapse maturation and function.

\section{Introduction}

Synaptogenesis is a complex process that leads to the precise alignment of presynaptic and postsynaptic specializations (Giagtzoglou et al., 2009). Ample in vitro studies have indicated that synaptic adhesion molecules play important roles in these processes (Scheiffele et al., 2000; Graf et al., 2004; Craig et al., 2006; Margeta and Shen, 2010). Although homotypic cell adhesion molecules such as Fas II are involved in the initial steps of synaptic recognition and adhesion (Ashley et al., 2005),

\footnotetext{
Received July 22, 2010; revised Sept. 12, 2010; accepted 0ct. 26, 2010.

This work was supported by Natural Science Foundation of China Grant 30370698, Key Grant of Ministry of Education Grant 03083, National Basic Research Program (973 Program) Grant 2005CB522501, Foundation for Excellent Doctoral Dissertation of Southeast University Grant YBJJ0520, the Open Research Fund of Jiangsu Key Laboratory of Neurodegeneration, and Canadian Institutes for Health Research Grant MOP 14143. G.L.B. is the recipient of a Tier I Canada Research Chair in Molecular and Developmental Neurobiology. We thank Drs. Zhengping Jia, William Trimble, and Mei Zhen for useful discussions and critical comments on this manuscript. We also thank Dr. Yongqing Zhang for technical assistance with the electrophysiology experiments, Dr. Xun Huang for technical assistance with gene targeting, Dr. Chunjie Zhao for helpful suggestions, and Jinjun Qian for help with bioinformatics. We are grateful to Dr. A. DiAntonio for anti-DGluR antibodies, Dr. N. Harden for anti-DPAK antibody, and the Bloomington Stock Center for providing Drosophila stocks.

Correspondence should be addressed to either of the following: Gabrielle L. Boulianne or Wei Xie, Toronto Medical Discovery Tower, 12th Floor, East Tower, 101 College Street, Toronto, Ontario, Canada M5G 1L7, E-mail: gboul@sickkids.ca; Wei Xie, The Key Laboratory of Developmental Genes and Human Disease, Ministry of Education, Institute of Life Science, Southeast University, 87 Dingjiaqiao Road, Nanjing 210009, China, E-mail: wei.xie@seu.edu.cn.

DOI:10.1523/JNEUROSCI.3854-10.2011

Copyright $\odot 2011$ the authors $\quad 0270-6474 / 11 / 310687-13 \$ 15.00 / 0$
}

heterotypic Neurexin and Neuroligin proteins are thought to be particularly important for establishing the asymmetry of the synapse, including the differential recruitment of presynaptic and postsynaptic proteins to their respective subcellular compartments (Scheiffele et al., 2000; Graf et al., 2004; Li et al., 2007a; Zeng et al., 2007).

Neuroligins constitute a family of proteins whose structural, biochemical, and cell biological characteristics have been well studied (Ichtchenko et al., 1995, 1996; Comoletti et al., 2003). In vertebrates, there are four neuroligin genes (Ichtchenko et al., 1995, 1996; Bolliger et al., 2001) that encode highly conserved proteins composed of a long extracellular sequence that is glycosylated, a single transmembrane domain, and a relatively short intracellular domain that includes a PDZ [postsynaptic density-95 (PSD-95)/Discs large (Dlg)/zona occludens-1] binding motif at the extreme $\mathrm{C}$ terminus (Irie et al., 1997). The large extracellular domain of Neuroligins exhibits high sequence similarity to acetylcholinesterase but lacks cholinesterase activity (Ichtchenko et al., 1995). Rather, the extracellular domain binds directly to its presynaptic partner Neurexin (Ichtchenko et al., 1995; Comoletti et al., 2003). The intracellular domain of Neuroligin can also interact with several postsynaptic molecules, including the PDZ-domain scaffolding protein PSD-95 and Shank (Irie et al., 1997; Iida et al., 2004; Meyer et al., 2004), which together are thought to recruit postsynaptic adaptor proteins to synaptic junctions. 
Early studies examining the function of Neurexin and Neuroligin in cell culture suggested that trans-synaptic interactions between the two proteins were essential for the formation of presynaptic and postsynaptic specializations (Scheiffele et al., 2000; Graf et al., 2004; Chih et al., 2005; Nam and Chen, 2005). More recent in vivo studies in mammals, however, suggest that Neurexin and Neuroligin are not required for synaptogenesis but rather promote the stabilization and development of newly formed synapses (Missler et al., 2003; Varoqueaux et al., 2006; Chubykin et al., 2007).

We have characterized the role of Drosophila neuroligin $2(d n l 2)$ in the development and function of the neuromuscular junction (NMJ). We show that $d n l 2$ is expressed in the ventral nerve cord (VNC) and at the NMJ. Null mutants in $d n l 2$ are viable but display locomotor defects. Importantly, we observe significant reductions in the number of synaptic boutons and in the abundance of postsynaptic receptors in $d n l 2$ mutants. We also find defects in postsynaptic densities and the subsynaptic reticulum (SSR), which are similar to those observed in immature synapses. $d n l 2$ mutants also exhibit alterations in synaptic transmission with increased stimulus-evoked transmitter release. Altogether, we show that, although $d n l 2$ is not essential for synapse formation, it is required for synapse maturation and neurotransmission.

\section{Materials and Methods}

Drosophila stocks. All stocks were grown at 22$25^{\circ} \mathrm{C}$ on standard medium. The wild-type (WT) Drosophila melanogaster strain used in this study was $w^{1118}$. The $24 \mathrm{~B}-\mathrm{Gal} 4$ line was obtained from the Kyoto stock center (stock number 101856) and C57-Gal4 line was a kind gift from V. Budnik (University of Massachusetts School of Medicine, Worcester, MA) (Budnik et al., 1996). UAS-dnl2 transgenic flies were generated by germ-line transformation using a construct containing the entire $d n l 2$ full-length cDNA in a pUAST vector. $\mathrm{Df}(2 \mathrm{~L})$ ED7007 (a deficiency that removes $d n l 2$ ) was obtained from the Bloomington Stock Center.

RNA interference (RNAi) transgenic flies (V44236 and V44237) against dnl2 were obtained from the Vienna Drosophila RNAi Center.

Genomic deletions of the Drosophila neuroligin 2 gene. Targeted knock-out (KO) of the $d n l 2$ genomic locus was generated as described previously (Gong and Golic, 2003). Briefly, two pairs of primers ( $5^{\prime}$-tgaGCGGCCGCGACCTGAGTAAGTCGGGATGG-3' and $5^{\prime}$-tgaGCGGCCGCGAGCTTCAATTGGCCCGCTGG-3'; 5' 'tgaGGCGCGCCAGTTGACGGCTTCGGGATTC- $3^{\prime}$ and $5^{\prime}$-tgaGGCGCGCCTCAAATCCAAATAGGACTCCAC-3') were used to amplify the upstream ( $5^{\prime}$ arm; -2924 to -9$)$ and downstream (3' arm; 1803-6079) fragments of the dnl2 gene from $w^{1118}$ DNA. These fragments were cloned into the pW25 vector (Drosophila Genomics Resource Center) and used for generating transformants. Transgenic flies were generated by embryo injection using standard methods (Spra-
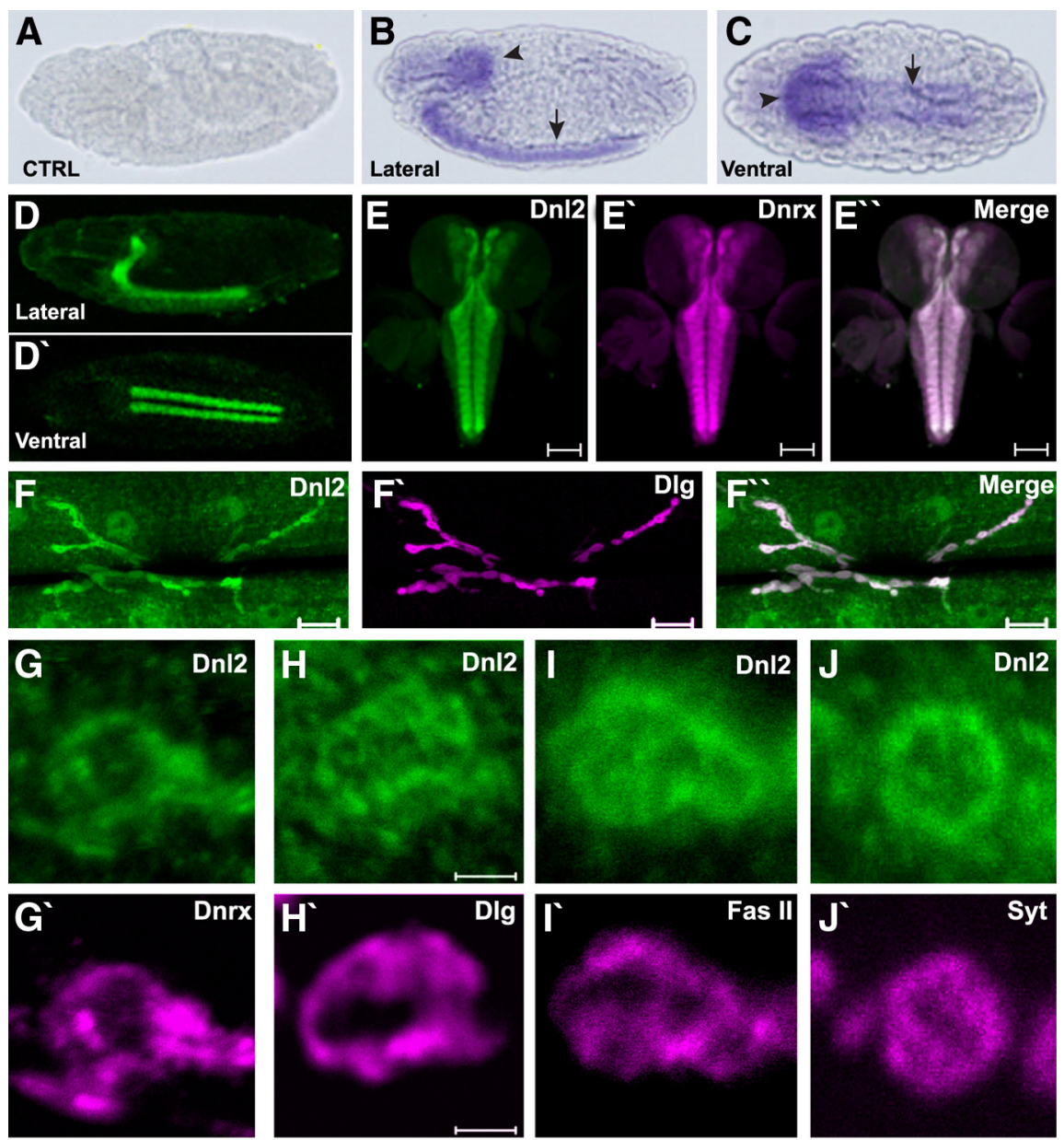
(arrowheads) and ventral nerve cord (arrow). D, $\boldsymbol{D}^{\prime}$, Lateral (D) and ventral ( $\left.\boldsymbol{D}^{\prime}\right)$ views of stage 16 embryos stained with anti-Dnl2 antibody confirming Dnl2 expression in the embryonic CNS. $\boldsymbol{E}-\boldsymbol{E}^{\prime \prime}$, Third-instar larval brain costained with anti-Dnl2 antibody (E) and anti-Dnrx $\left(\boldsymbol{E}^{\prime}\right)$ showing colocalization of these two proteins $\left(\boldsymbol{E}^{\prime \prime}\right) . \boldsymbol{F}-\boldsymbol{F}^{\prime \prime}$, Double staining of wide-type third-instar larval NMJ on muscles 6/7 with anti-Dnl2 $(\boldsymbol{F})$ and anti-Dlg ( $\boldsymbol{F}^{\prime}$, which labels glutamatergic type I boutons), showing that Dnl2 is highly expressed double labeled for DnI2 (G-J) and the synaptic marker $\operatorname{Dnrx}\left(\boldsymbol{G}^{\prime}\right)$, Dlg $\left(\boldsymbol{H}^{\prime}\right)$, Fas II $\left(\boldsymbol{I}^{\prime}\right)$, or Syt $\left(\boldsymbol{J}^{\prime}\right)$ showing colocalization of Dnl2 with these proteins. Scale bars: $\boldsymbol{E}-\boldsymbol{E}^{\prime \prime}, 50 \mu \mathrm{m} ; \boldsymbol{F}-\boldsymbol{F}^{\prime \prime}, 20 \mu \mathrm{m} ; \boldsymbol{H}-\boldsymbol{H}^{\prime \prime}, 1 \mu \mathrm{m}$.

dling and Rubin, 1982). Virgin female donor flies bearing the targeting construct on the third chromosome were crossed to $y w$; P[70 FLP]P[70ISceI]/TM6 (Bloomington Stock Center \#6935) flies and the 3-d-old progeny were heat shocked for $60 \mathrm{~min}$ at $38^{\circ} \mathrm{C}$ to induce homologous recombination. A total of 18 independent $d n l 2^{\mathrm{KO}}$ alleles with homologous recombination events between the donor DNA fragment and the $d n l 2$ locus were identified by genomic PCR screening. Two independent lines, $d n l 2^{K O 70}$ and $d n l 2^{K O 17}$, were further confirmed by using Western blot analysis and used for the present study.

Antibody production, Western blotting, and immunoprecipitation. Rabbit polyclonal antibodies and mouse monoclonal antibodies against Dnl2 were generated using a recombinant protein containing the cytoplasmic region of Dnl2 fused with a His6 tag at the $\mathrm{N}$ terminus. The serum was 

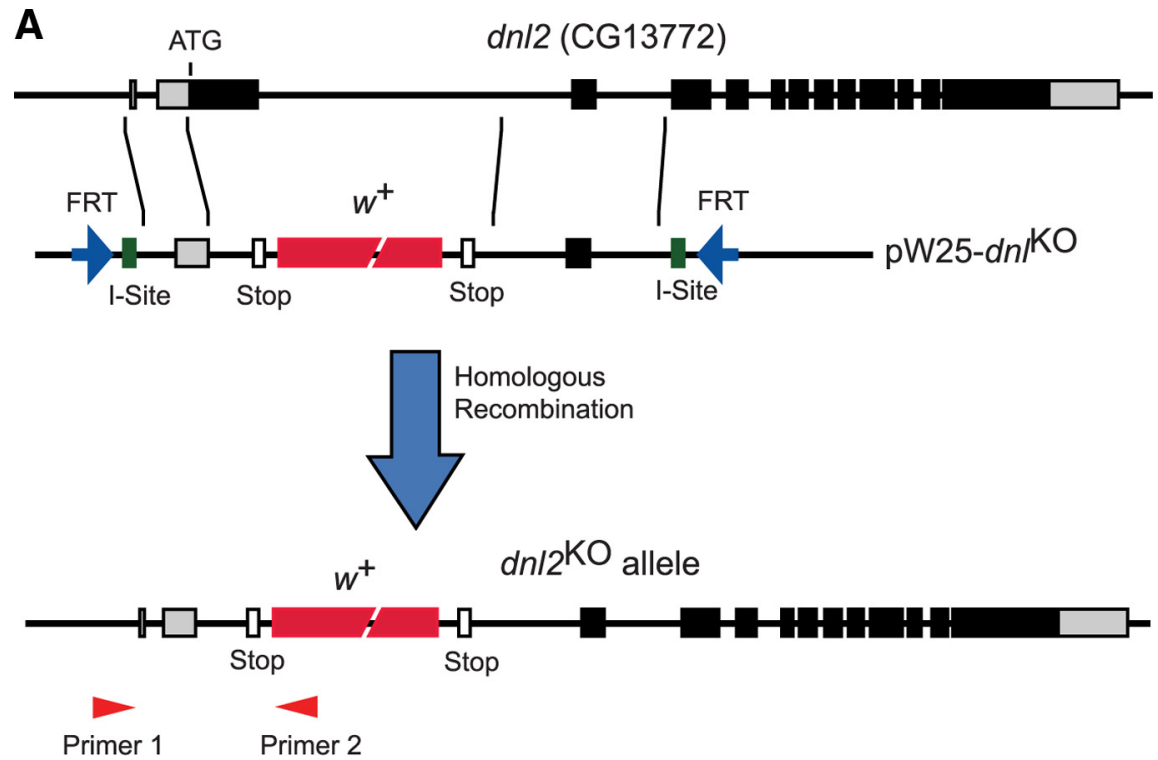

B

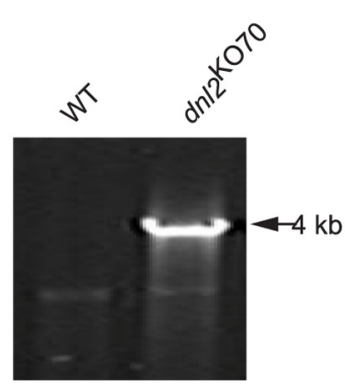

C

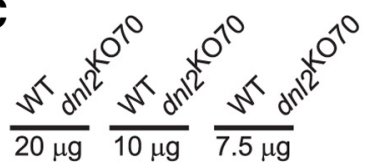

D

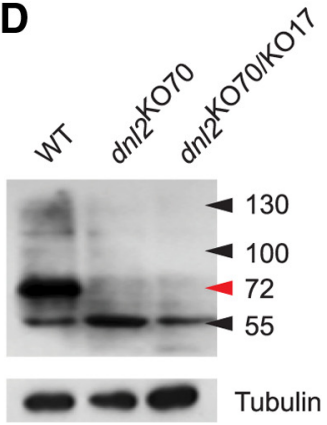

Figure 2. Targeted inactivation of $d n / 2$ by homologous recombination. $A$, The structure of the $d n / 2$ locus, targeting vector, and targeted locus are shown. Positions of primers used for PCR screening of targeted mutant flies in $B$ are shown at the bottom of $A$. $B, P C R$ analysis of genomic DNA from WT and $d n / 2^{K 070}$ flies. A PCR product with a predicted size of $4.0 \mathrm{~kb}$ is generated only with genomic DNA from mutant flies because primer 1 is located outside the $5^{\prime}$ arm of the targeting vector and primer 2 is within the white gene. C, Northern blot analysis of total RNA isolated from the adult head using a dn/2 probe showing the predicted full-length $4.8 \mathrm{~kb}$ mRNA in WT but not in $d n / 2^{K 070}$ flies. D. Western blot analysis of adult head extracts using anti-Dnl2 showing the expression of a $70 \mathrm{kDa}$ protein in WT but not in homozygous $d n 12^{K 070}$ or $\mathrm{dn} / 2^{\mathrm{KOT0/K017}}$ mutant flies.

affinity purified using a column packed with the same recombinant protein fused to glutathione $S$-transferase and used for Western blot analysis and immunostaining at the indicated dilutions.

Western blotting analysis was performed as described previously (Edery et al., 1994; Zeng et al., 2007). In brief, adult heads were homogenized with lysis buffer and centrifuged at $13,000 \mathrm{rpm}$ at $4^{\circ} \mathrm{C}$ for $10 \mathrm{~min}$, and the supernatant was collected. The protein lysates were electrophoresed on an $8 \%$ SDS-PAGE gel and electrotransferred onto polyvinylidene difluoride membranes. Immobilized proteins on the membrane were probed with the rabbit polyclonal antibody to Dnl2 (1:1000 dilution) and then incubated with HRP-conjugated secondary antibodies. The same blots were probed with a mouse monoclonal antibody to Tubulin $(1: 10,000$ dilution) as a loading control [E7; Developmental Studies Hybridoma Bank (DSHB)]. The targeted proteins were visualized with Qentix Western signal enhancer and SS West Pico Substrate detection (Pierce). For immunoprecipitation, adult heads were lysed in radioimmunoprecipitation assay (RIPA) buffer with protease inhibitor (Roche). A total of $25 \mathrm{ml}$ of $50 \% \mathrm{RIPA} / 50 \%$ protein A-Sepharose plus $3 \mathrm{mg}$ of antibody [anti-Dnl2 or anti-Drosophila neurexin (Dnrx)] was used for overnight incubation at $4^{\circ} \mathrm{C}$. Protein A beads were washed and resuspended in SDS loading buffer (with $100 \mathrm{~mm}$ DTT) and analyzed by SDS-PAGE and immunoblotting.

In situ hybridization and immunocytochemistry. The sequence encoding the first 200 amino acids of the $d n l 2$ gene was PCR amplified and cloned into pBluescript SKII (-) (Stratagene). Digoxigenin (DIG)- labeled sense and antisense RNA probes were generated in vitro with a DIG labeling kit (Roche Applied Science) using linearized pBluescript SKII (-)-Neuroligin $2(1-200)$ as templates. Whole-mount in situ hybridization was performed according to standard procedures (Vosshall et al., 2000).

Preparation and immunostaining of wholemount embryos and dissected wandering thirdinstar larvae were performed as described previously (Zeng et al., 2007). For immunostaining experiments, the primary antibodies used included the following: rabbit anti-Dnl2 (1:100), mouse anti-Dnl2 6D5 (1:10), anti-Bruchpilot (BRP) (nc82; 1:25; DSHB), monoclonal antiDLG 4F3 (1:500; DSHB), monoclonal anti-Fas II (1:25; DSHB), anti-GluRIIA (1:50; DSHB), monoclonal anti-synaptotagmin (Syt) (1:50; DSHB), rabbit anti-Drosophila p21-activated kinase (DPAK) (1:2000; gift from N. Harden, Simon Fraser University, Burnaby, BC, Canada) (Harden et al., 1996), and rabbit anti-GluRIII and rabbit anti-GluRIIB (1:2000; gifts from A. DiAntonio, Washington University, St. Louis, MO) (Marrus et al., 2004). The secondary antibodies used were conjugated to tetramethylrhodamine isothiocyanate or FITC (1:300; Jackson ImmunoResearch).

Imaging and analysis. Images were collected and analyzed on a Carl Zeiss confocal station using the LSM510 software. Quantification of bouton numbers was performed at muscles $6 / 7$ and muscle 4 of abdominal segment 3 . The total boutons at NMJ6/7 and type Ib boutons at NMJ4 were visualized by staining of body wall muscle reparations with anti-HRP antibodies and anti-Dlg. Branches were defined as sections of neurites containing at least two boutons and extending from the principal axis of the axon that runs along the boundary between the 6 and 7 muscles.

For comparisons of fluorescent intensities across genotypes, samples from different genotypes were dissected and fixed under the same conditions, processed in the same vial, and imaged with identical settings. Individual synapses were optically sectioned at $0.2 \mu \mathrm{m}(18-25$ sections per synapse) using a piezo-electric driven $\mathrm{z}$-drive controlling the position of a Carl Zeiss $63 \times$ oil-immersion objective. The intensity of immunostaining was quantified as follows: a twodimensional projection of the maximal fluorescence at the NMJ (muscle 4) was created from a series of $0.2 \mu \mathrm{m}$ synaptic sections. The average fluorescence was calculated over the entire synaptic area. For analyzing DGluR levels, synaptic areas were defined as delimited by HRP immunoreactivity, and the staining intensity within this area was averaged.

Electron microscopy. The methods for EM of third-instar larvae NMJ $6 / 7$ used in this study were described previously by Schmidt et al. (2008). In brief, third-instar larvae body wall muscles were rapidly dissected in physiological Ringer's solution (in mM: $160 \mathrm{NaCl}, 3 \mathrm{MgCl}$, and 12 HEPES). The dissected third-instar larvae (NMJ 6/7; segment A3) were first fixed for $4 \mathrm{~h}$ at $4{ }^{\circ} \mathrm{C}$ in $4 \%$ glutaraldehyde, $2 \mathrm{~mm} \mathrm{CaCl}_{2}$, and $4 \mathrm{~mm}$ $\mathrm{MgCl}_{2}$ in $0.1 \mathrm{M}$ sodium cacodylate buffer, $\mathrm{pH}$ 7.2. The samples were then washed four times with $0.1 \mathrm{~m}$ sodium cacodylate buffer, $\mathrm{pH} 7.2$, and postfixed for $1.5 \mathrm{~h}$ with wash buffer containing $1 \%$ osmium tetroxide. After three brief washing steps in distilled water, the samples were stained en bloc for $1 \mathrm{~h}$ on ice with $1 \%$ uranyl acetate in distilled water. After a brief wash with distilled water, samples were dehydrated at room temperature with increasing ethanol concentrations, infiltrated in Epon resin (first in 100\% EtOH/Epon at 1:1, for 30 and $90 \mathrm{~min}$, and then in $100 \%$ Epon overnight), and embedded in three successive steps at $30^{\circ} \mathrm{C}, 45^{\circ} \mathrm{C}$, 
Table 1. Synaptic transmission is elevated in $\mathrm{dn} / 2^{\mathrm{KO} 0}$ mutants

\begin{tabular}{lccccc}
\hline & $w^{1118}$ & & & $d n / 2^{\text {KO70 }}$ & \\
\cline { 2 - 3 } & Low calcium & High calcium nn & & Low calcium & High calcium \\
\hline mEJP amplitude $(\mathrm{mV})$ & $0.95 \pm 0.05$ & $0.95 \pm 0.05$ & & $0.89 \pm 0.04$ & $0.96 \pm 0.05$ \\
mEJP frequency $(\mathrm{Hz})$ & $1.75 \pm 0.3$ & $1.4 \pm 0.1$ & & $1.27 \pm 0.15$ & $1.6 \pm 0.1$ \\
EJP amplitude $(\mathrm{mV})$ & $20.9 \pm 2.1$ & $39.8 \pm 1.2$ & & $28.3 \pm 2.3$ & $46.9 \pm 2.3$ \\
Rise time (ms) & $7.2 \pm 0.4$ & $8 \pm 0.3$ & & $4.9 \pm 0.25$ & $6.1 \pm 0.14$ \\
Decay time (ms) & $19.3 \pm 1.2$ & $18.8 \pm 1.1$ & & $10.8 \pm 0.9$ & $14.7 \pm 0.5$ \\
\hline
\end{tabular}

Values are listed as the mean \pm SEM. Low calcium, $0.2 \mathrm{~mm}$; High calcium, $0.8 \mathrm{~mm}$.

and $60^{\circ} \mathrm{C}$, each lasting for $24 \mathrm{~h}$. The samples were then trimmed, and series of $80-90 \mathrm{~nm}$ ultrathin sections were cut with a $35^{\circ}$ diamond knife (Diatome) on a Reichert Ultracut Ultramicrotome (Leica) and mounted on Formvar-coated grids. The sections were finally stained in uranyl acetate and lead citrate. Micrographs were obtained with a Philips EM 301 transmission electron microscope.

Electrophysiology. Conventional intracellular recordings were used for assessing NMJ neurotransmission (Jan and Jan, 1976). Wandering thirdinstar larvae were dissected in $\mathrm{Ca}^{2+}$-free HL3.1 saline, gut and fat were removed, and the body wall was spread out to expose the nerve and muscle. Microelectrodes $(20-50 \mathrm{M} \Omega$ ) were pulled from the borosilicate glass (WPI) with a glass puller (P-2000; Sutter Instruments) and filled with a 50:50 mix of $3 \mathrm{~m} \mathrm{KCl}$ and $3 \mathrm{~m}$ potassium acetate. Recordings were performed at room temperature with an Axoclamp 2B amplifier (Molecular Devices) in bridge mode. Recording data were digitized with a Digitizer 1322A (Molecular Devices) and collected by pClamp 9.1 (Molecular Devices) software. For excitatory junction potential (EJP) recordings, the segmental nerve was cut, and the free end was drawn into a microelectrode and stimulated with a Grass S48 stimulator (Astro-Grass) at $0.3 \mathrm{~Hz}$ with suprathreshold stimulating pulse, and 25-30 EJPs in NMJ 6 in A3 segment were recorded in each animal. Miniature EJPs (mEJPs) were recorded for $120 \mathrm{~s}$ after EJP recordings. Data were processed with miniAnalysis (Synaptosoft) software and statistically evaluated with SigmaPlot software. Only the recordings with resting membrane potentials ranging from -55 to $-65 \mathrm{mV}$ were used for analysis. Paired-pulse experiments were performed by delivering pairs of stimuli at varying interstimulus intervals in $0.2 \mathrm{~mm}$ calcium. The paired-pulse ratio was calculated as the amplitude of the second EJP (test pulse) divided by the amplitude of the first EJP (conditioning pulse).

Statistical analysis. In all experiments, analyses, and whenever possible, experiments were performed blind with respect to the genotypes used. All the averaged data in this study were reported as mean \pm SEM. $p<$ 0.05 was considered to be significant. Statistical significance was determined using either Student's tests for comparisons of two groups or ANOVAs followed by the appropriate post hoc test for comparisons of multiple groups. $p$ values and the test used are indicated in figure legends.

\section{Results}

Identification of the Drosophila Neuroligin 2 gene

Analysis of the Drosophila melanogaster genome revealed the presence of four neuroligin-like genes (CG13772, CG34127, CG34139, and CG31146). All four of the putative neuroligin genes encode proteins that share significant amino acid similarity with vertebrate Neuroligin and a similar predicted protein structure; however, based on protein sequence alignments, the four Drosophila neuroligins and mammalian neuroligins evolved from a common ancestor (supplemental Fig. 1, available at www.jneurosci.org as supplemental material). As such, we cannot draw a direct correlation between any one Drosophila neuroligin and the mammalian neuroligins. We had previously identified a full-length cDNA clone from a Drosophila brain cDNA library that corresponds to the CG13772 gene, which was submitted to flybase as dneuroligin ( $d n l)$ (http://www.ncbi.nlm.nih. gov/nuccore/7716609?report=genbank). A recent unbiased screen for genes affecting NMJ structure, however, identified CG31146 and named that homolog Drosophila neuroligin 1 (Banovic et al., 2010). The present study examines the role of the
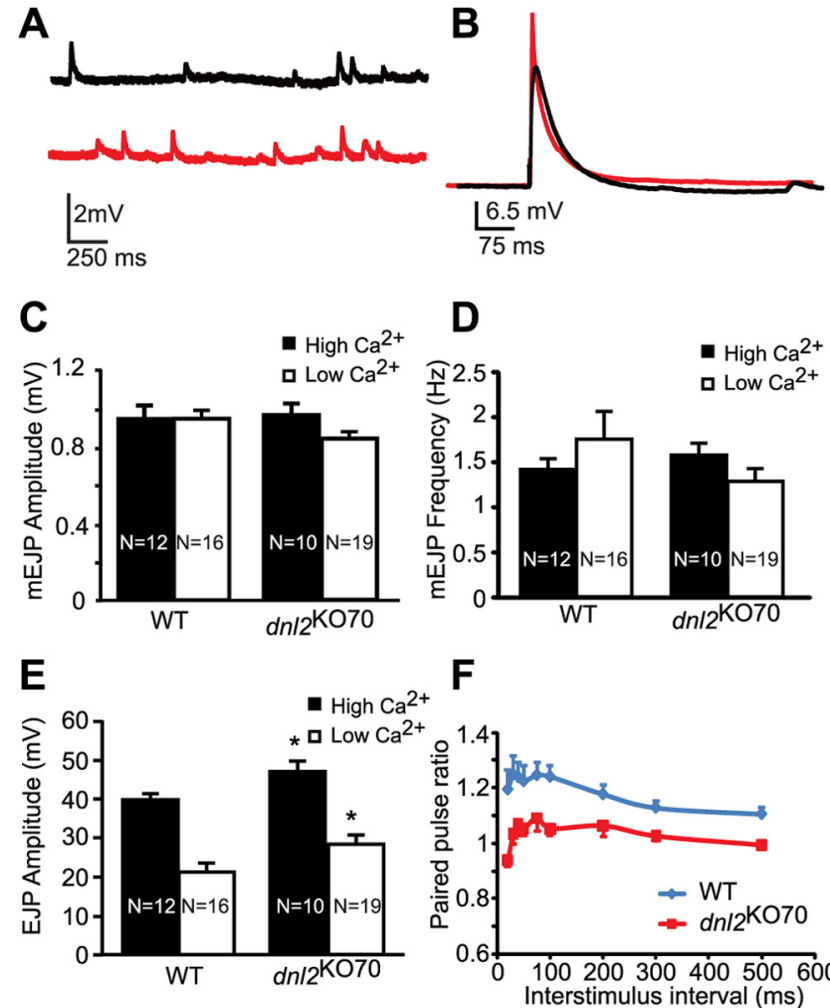

$\mathbf{F}$
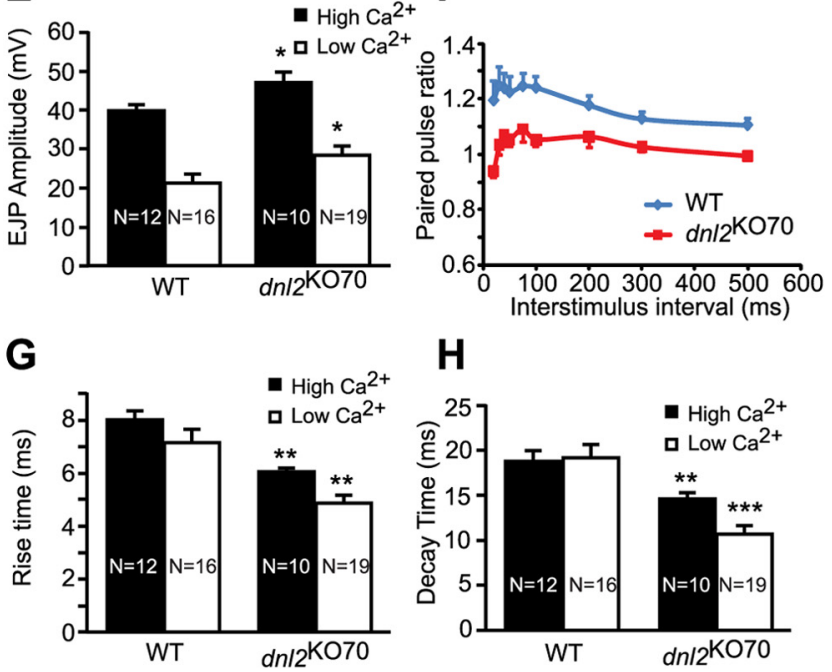

H

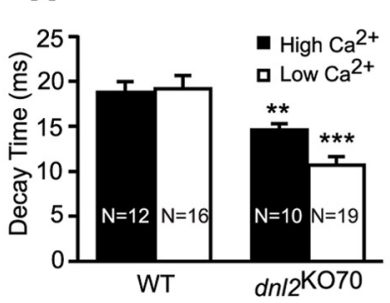

Figure 3. Enhanced synaptic transmission at NMJs in $d n / 2$ mutants. $\boldsymbol{A}$, Representative traces of mEJPs in WT (top row) and $d n / 2^{K 070}$ (bottom row) mutants recorded from muscles 6/7 in abdominal segment 3. $\boldsymbol{B}$, Representative traces of stimulus-evoked EJPs in muscles $6 / 7$ in WT (black trace) and $d n / 2^{K O 70}$ (red trace) mutants recorded in $0.8 \mathrm{~mm}$ calcium. $\boldsymbol{C}, \boldsymbol{D}$, No significant differences were observed in either the amplitudes $(\boldsymbol{C})$ or frequencies $(\boldsymbol{D})$ of mEJPs between WT and $d n 12^{K O 70}$ mutants recorded in either high $(0.8 \mathrm{~mm})$ or low $(0.2 \mathrm{~mm})$ calcium. $\boldsymbol{E}$, Stimulusevoked EJP amplitudes were significantly increased in $d n 12^{K 070}$ mutants $\left({ }^{*} p<0.05\right.$, Student's $t$ test) in both high $(0.8 \mathrm{~mm})$ and low $(0.2 \mathrm{~mm})$ calcium. $\boldsymbol{F}$, Pairs of stimuli were delivered at varying interstimulus intervals in $0.2 \mathrm{~mm}$ calcium, and the paired-pulse ratio was calculated as the amplitude of the second EJP divided by the amplitude of the first EJP. dnI2 ${ }^{K 070}$ mutants showed significantly less facilitation than the controls at all interstimulus intervals tested. $\mathbf{G}, \boldsymbol{H}$, Both the rise times $(\boldsymbol{G})$ and the decay times $(\boldsymbol{H})$ of stimulus-evoked EJPs were shorter in $d n / 2^{K O 70}$ mutants in both high $(0.8 \mathrm{~mm})$ and low $(0.2 \mathrm{~mm})$ calcium. ${ }^{* *} p<0.01$ versus WT, ${ }^{* *} p<0.001$ versus WT, Student's $t$ test.

CG13772 homolog originally named dneuroligin, which we now term Drosophila neuroligin $2(d n l 2)$ to avoid confusion. The $d n l 2$ gene is located on the left arm of the second chromosome at cytological position 27C3-4 and is composed of 13 exons and 12 introns. $d n l 2$ encodes a 1248 amino acid long protein with a predicted molecular weight of $137 \mathrm{kDa}$. Similar to vertebrate neuroligins, Dnl2 is also predicted to comprise three distinct regions: an $\mathrm{N}$-terminal extracellular acetylcholinesterase-like domain, a single transmembrane region, and a C-terminal cytoplasmic region with a conserved PDZ binding motif (supplemental Fig. S1, available at www. jneurosci.org as supplemental material). The extracellular domain also contains several putative $N$-glycosylation sites and a serine/ 

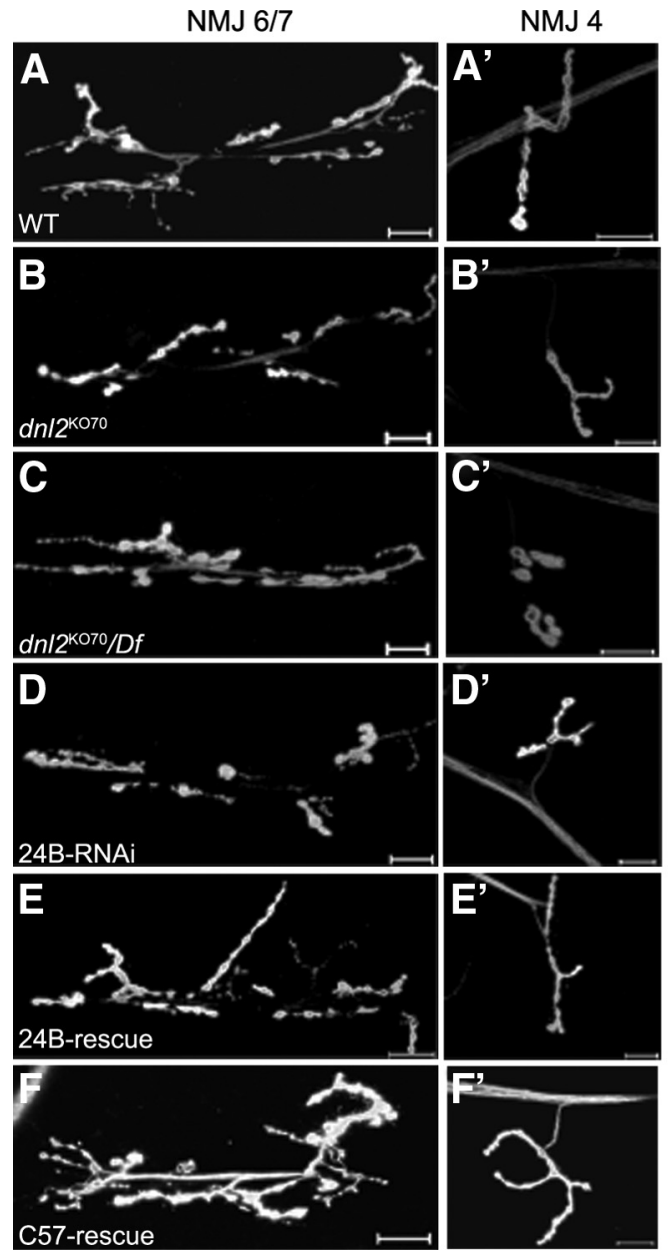

\section{G}
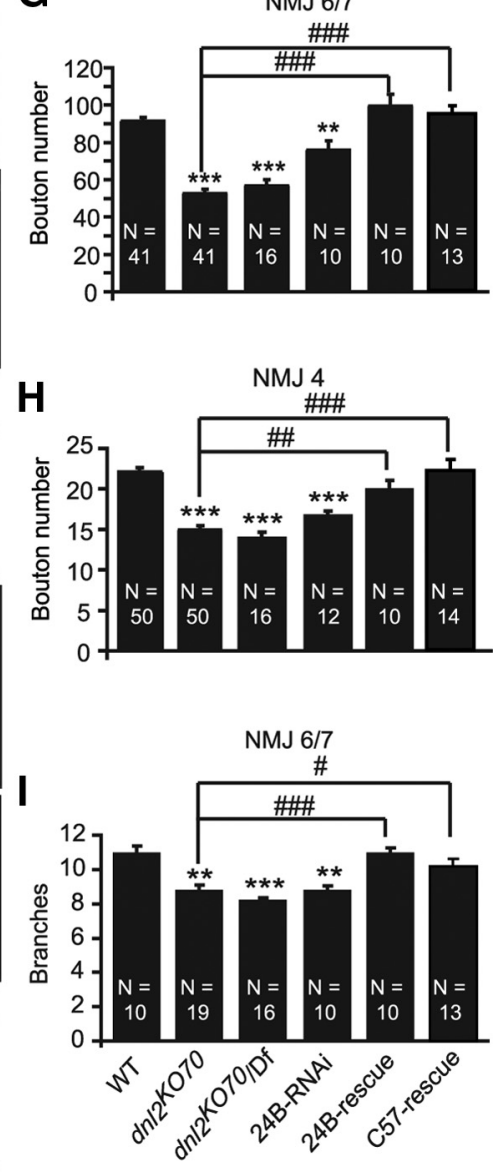

Figure 4. Reduced synaptic boutons at larval NMJs in $d n / 2$ mutants. $\boldsymbol{A}-\boldsymbol{F}^{\prime}$, NMJs at muscle $6 / 7(\boldsymbol{A}-\boldsymbol{F})$ and muscle $4\left(\boldsymbol{A}^{\prime}-\boldsymbol{F}^{\prime}\right)$ of larval abdominal segment 3 labeled with anti-HRP and anti-Dlg showing reduced NMJ expansion and fewer boutons in $d n / 2$ null mutants $d n / 2^{K O 70}\left(\boldsymbol{B}, \boldsymbol{B}^{\prime}\right), d n / 2^{K 070} / \mathrm{Df}\left(\boldsymbol{C}, \boldsymbol{C}^{\prime}\right)$, and transgenic mutants expressing $d n / 2$ RNAi driven by $24 \mathrm{~B}-\mathrm{Gal} / 4\left(\boldsymbol{D}, \boldsymbol{D}^{\prime}\right)$ compared with WT flies $\left(\boldsymbol{A}, \boldsymbol{A}^{\prime}\right)$. The deficits in mutant $d n / 2^{K 070}$ is rescued by muscle expression of UAS-WT $d n / 2$ CDNA with either 24B-Gal4 $\left(\boldsymbol{E}, \boldsymbol{E}^{\prime}\right)$ or $\boldsymbol{C 5 7 - G a l} 4\left(\boldsymbol{F}, \boldsymbol{F}^{\prime}\right)$. Scale bars, $20 \mu \mathrm{m} . \boldsymbol{G}, \boldsymbol{H}$, Summary graphs showing significant reduction in the numbers of total boutons at NMJ 6/7 (G) and type lb bouton at NMJ4 $(\boldsymbol{H})$ in both null mutants and dn/2 RNAi transgenic flies compared with WT control. $\boldsymbol{I}$, Summary graph showing decreased synaptic branching in $d n / 2$ null mutants and $d n / 2$ RNAi transgenic flies. Note the reduced branching and bouton numbers in $d n / 2$ null mutants and $d n / 2$ RNAi transgenic flies are rescued by muscle expression of a UAS- $d n / 2$ CDNA with either 24B-Gal4 (24B-rescue) or (57-Gal4 (C57-rescue). ${ }^{* *} p<0.01$ versus WT, ${ }^{* * *} p<0.001$ versus WT, ${ }^{\# \#} p<0.01$

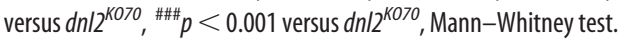

threonine-rich region for potential O-glycosylation between the acetylcholinesterase-like domain and the transmembrane domain that may affect neurexin binding (Comoletti et al., 2003).

$d n l 2$ is expressed in central neurons and at glutamatergic neuromuscular junctions

To determine the expression of $d n l 2$, we first performed in situ hybridization using DIG-labeled RNA probes. In early embryonic stages, $d n l 2$ transcripts were detected uniformly throughout the embryo (data not shown). However, by stage $14, d n l 2$ mRNA was primarily restricted to the brain and VNC (Fig. $1 B, C$ ). To verify these results at the protein level, we developed an affinitypurified monoclonal antibody against the $\mathrm{C}$ terminus of Dnl2 and performed immunostaining. The specificity of this antibody was confirmed by using $d n l 2$ null mutants (supplemental Fig. S2, available at www.jneurosci.org as supplemental material). Consistent with in situ experiments, Dnl2 protein was enriched in neuropil regions of the embryonic brain and ventral nerve cord (Fig. 1D). High levels of Dnl2 were also observed in the brain and
VNC of third-instar larvae in which it colocalized with Drosophila neurexin (Dnrx) (Fig. 1E). Dnl2 expression in the CNS was widespread and uniform and did not appear to be preferentially localized with any specific neurotransmitter or neuro-hormone. We also detected Dnl2 staining in the abdominal muscles of third-instar larvae, with more concentrated staining at NMJs (Fig. $1 F$ ). To more precisely determine the localization of Dnl2 within boutons, we compared Dnl2 staining with an assortment of characterized synaptic markers. Similar to our results in the CNS, Dnl2 strongly colocalized at the NMJ with neurexin (Fig. $\left.1 G^{\prime \prime}\right)$. We also observed strong colocalization of Dnl2 with the postsynaptic PSD95-like protein Dlg (Fig. $\left.1 H^{\prime \prime}\right)$, the neural cell adhesion molecule homolog Fas II (Fig. $1 I^{\prime \prime}$ ), and the presynaptic vesicle marker Syt (Fig. $1 J^{\prime \prime}$ ). Together, these results suggest that Dnl2 is strongly expressed at both developing and mature synapses.

\section{Targeted disruption of the Drosophila dnl2 gene}

To investigate the in vivo functions of $d n l 2$, we generated $d n l 2$ null mutants using gene targeting through ends-out homologous recombination (Gong and Golic, 2003) (see Materials and Methods). Briefly, the targeting construct consisted of a mini-white marker gene flanked by 2.9 and $4.3 \mathrm{~kb}$ genomic sequences surrounding the first intron of $d n l 2$ (Fig. $2 A$ ). Homologous recombination between the donor DNA and endogenous $d n l 2$ locus resulted in a null allele by replacing a $2 \mathrm{~kb}$ fragment (from the start codon ATG to the middle of the first intron of the $d n l 2$ gene) with the mini-white transgene. Eighteen independent $d n l 2$ knock-out $\left(d n l 2^{\mathrm{KO}}\right)$ lines were identified by PCR analysis, and the absence of $d n l 2$ gene expression in these lines was confirmed by Northern and Western blotting analysis (Fig. $2 B-D$ ). Of note, in WT flies, the predominant form of Dnl2 that we detected had an apparent molecular weight of $70 \mathrm{kDa}$. This was much smaller than the predicted size, suggesting that the protein may undergo extensive processing. We also detected a similar $70 \mathrm{kDa}$ protein using two additional anti-Dnl2 antibodies that recognized either the C-terminal portions of Dnl2 or the central domain (data not shown). In all cases, the $70 \mathrm{kDa}$ form of Dnl2 was detected in extracts from wild-type flies and was completely absent in $d n l 2^{\mathrm{KO}}$ mutant lines, indicating that the antibody is specific for Dnl2 and that the mutants are protein null. The $d n l 2^{\mathrm{KO}}$ lines were viable and fertile with no detectable abnormalities in body size or morphology. However, locomotor tests showed that the mutant larvae had significantly reduced locomotor activity (supplemental Fig. S3, available at www.jneurosci.org as supplemental material), which may 
Table 2. dnI2 ${ }^{\mathrm{K} 070}$ NMJs have numerous presynaptic and postsynaptic defects

\begin{tabular}{|c|c|c|c|c|c|c|}
\hline & $w^{1118}$ & $d n / 2^{K 070}$ & $d n / 2^{K 070} / D f$ & 24B-RNAi & $24 \mathrm{~B}-$ rescue & C57-rescue \\
\hline \multicolumn{7}{|l|}{ NMJ morphology } \\
\hline $\mathrm{m} 6 / 7$ boutons & $91.4 \pm 1.8$ & $52.5 \pm 2.4$ & $56.7 \pm 3.3$ & $76 \pm 5$ & $99.6 \pm 6.1$ & $89.5 \pm 4.1$ \\
\hline $\mathrm{m} 4$ boutons & $22.1 \pm 0.6$ & $14.9 \pm 0.5$ & $13.9 \pm 0.8$ & $16.6 \pm 0.6$ & $19.9 \pm 1.2$ & $21.8 \pm 1.4$ \\
\hline Branch points & $10.9 \pm 0.4$ & $8.7 \pm 0.3$ & $8.1 \pm 0.2$ & $8.7 \pm 0.3$ & $10.9 \pm 0.4$ & $9.8 \pm 0.4$ \\
\hline \multicolumn{7}{|l|}{ Active zones } \\
\hline Brp clusters & $19.1 \pm 0.8$ & $22.7 \pm 0.9$ & $22.8 \pm 0.9$ & ND & ND & ND \\
\hline Brp intensity (\%) & $100 \pm 6.6$ & $110.1 \pm 10$ & $117.1 \pm 8.1$ & ND & ND & ND \\
\hline DPAK clusters & $18.9 \pm 0.8$ & $22.5 \pm 0.8$ & $22.9 \pm 0.9$ & ND & ND & ND \\
\hline cluster size $\left(\mu \mathrm{m}^{2}\right)$ & $1.4 \pm 0.07$ & $1 \pm 0.04$ & $1.1 \pm 0.06$ & ND & ND & ND \\
\hline DPAK intensity (\%) & $100 \pm 8.3$ & $94.5 \pm 8.4$ & $99.2 \pm 6.5$ & ND & ND & ND \\
\hline \multicolumn{7}{|l|}{ Ultrastructure } \\
\hline T-bars & $1.3 \pm 0.2$ & $2 \pm 0.2$ & $2.5 \pm 0.3$ & ND & $1.2 \pm 0.3$ & ND \\
\hline SSR thickness(nm) & $708.1 \pm 57.1$ & $473.2 \pm 21.2$ & $487.3 \pm 24.7$ & ND & $721.1 \pm 47.7$ & ND \\
\hline PSD length (nm) & $617.2 \pm 16.6$ & $444.8 \pm 12.3$ & $472.7 \pm 16.4$ & ND & $632.8 \pm 26$ & ND \\
\hline \multicolumn{7}{|l|}{ GluR staining } \\
\hline GluRIII (\%) & $100 \pm 4.7$ & $60.6 \pm 3.6$ & $49.8 \pm 6$ & $80.2 \pm 5.8$ & $91.8 \pm 7.6$ & $122 \pm 6.3$ \\
\hline GluRIIA (\%) & $100 \pm 6.1$ & $124 \pm 7.1$ & $121.9 \pm 6.6$ & $122.8 \pm 7$ & $124.3 \pm 8$ & $130.4 \pm 9.6$ \\
\hline GluRIIB (\%) & $100 \pm 5.9$ & $62.7 \pm 2.6$ & $58.4 \pm 3.5$ & $61.6 \pm 1.3$ & $77.6 \pm 5.3$ & $20.1 \pm 0.8$ \\
\hline
\end{tabular}

$\mathrm{m} 6 / 7$ boutons, Total number of $\mathrm{lb}$ and $\mathrm{l} \mathrm{s}$ boutons on muscles 6 and 7 in abdominal segment $3 ; \mathrm{m} 4$ boutons, total number of $\mathrm{lb}$ boutons on muscle 4 in abdominal segment 3 ; Branch points, number of neurites containing at least two bouton and extending from the principal axis of the axon that runs along the boundary between the 6 and 7 muscles; Brp and DPAK clusters, number of Brp- or DPAK-positive clusters per bouton. Brp and DPAK intensity, staining intensity of Brp and DPAK clusters, respectively, expressed as a percentage of the WT staining intensity; T-bars, number of T-bars per bouton; SSR thickness and PSD length, morphometric measurements of the thickness of the SSR and PSD; GluR staining, intensity of GluR subunit staining expressed as a percentage of the WT value; ND, no data for that genotype.

suggest defects in synaptic function at the NMJ and/or in the CNS.

\section{Altered synaptic transmission in $d n l 2$ mutants}

Previous studies in mice have shown functional defects in neuroligin loss-of-function mutants (Varoqueaux et al., 2006; Chubykin et al., 2007). To investigate a potential synaptic basis for the locomotor deficit observed in $d n l 2$ mutants, we examined synaptic transmission at the NMJ. We first examined spontaneous and evoked transmission under approximately physiological conditions ( $0.8 \mathrm{~mm}$ calcium). Under these conditions, we found that neither the amplitude nor frequency of spontaneously occurring mEJPs were significantly different in $d n l 2$ mutants (Table 1, Fig. $3 A, C, D)$. In contrast, however, the amplitude of stimulusevoked EJPs showed a small but significant increase in $d n l 2 \mathrm{mu}-$ tants (Table 1, Fig. $3 B, E$ ). Similar results were seen in NMJs of $d n l 2^{K O 70}$ flies balanced over a deficiency that covers the neuroligin 2 gene region $\left(\mathrm{mEJP}\right.$ amplitude, $d n l 2^{K O 70} / \mathrm{Df}=0.91 \pm 0.5$ $\mathrm{mV}$; mEJP frequency, $d n l 2^{K O 70} / \mathrm{Df}=2 \pm 0.1 \mathrm{~Hz}, p<0.05 \mathrm{vs} \mathrm{WT}$; EJP amplitude, $d n l 2^{K O 70} / \mathrm{Df}=46 \pm 1 \mathrm{mV}, p<0.01$ vs WT). Because the postsynaptic response to a single vesicle of transmitter (mEJP amplitude) is not significantly altered in $d n l 2$ mutants, the results suggest that more transmitter is released from $d n l 2$ mutant terminals after stimulation. Given the large size of the EJPs recorded in $0.8 \mathrm{~mm}$ calcium, we wondered whether larger differences in transmitter release may be masked by either nonlinear summation of the EJPs or saturation of the postsynaptic receptors. To address this issue, we reexamined spontaneous and stimulus-evoked transmitter release under conditions of lower transmitter release probability $(0.2 \mathrm{~mm}$ calcium). Under these conditions, we still observed no significant difference in mEJP amplitude or frequency (Table 1, Fig. 3C,D). As expected, stimulus-evoked transmitter release was significantly reduced in both control and $d n l 2^{K O 70}$ mutants at $0.2 \mathrm{mM}$ calcium compared with $0.8 \mathrm{~mm}$ calcium (Table 1 , Fig. $3 E)$. As seen in high $(0.8 \mathrm{~mm})$ calcium, however, stimulus-evoked EJP amplitude was significantly elevated in $d n l 2^{K O 70}$ mutants in low $(0.2 \mathrm{mM})$ calcium (Table 1, Fig. 3E). Furthermore, the relative increase in EJP amplitudes observed in $d n l 2^{K O 70}$ mutants in low calcium was not different from the increase observed in high calcium, suggesting that the small difference between control and $d n l 2^{K O 7 O}$ is not attributable to a saturation of either the release machinery or the postsynaptic receptors.

The above results may be indicative of an increase in transmitter release probability. To determine whether transmitter release probability is altered in $d n l 2$ mutants, we examined paired-pulse plasticity. Pairs of stimuli were delivered in low calcium $(0.2 \mathrm{~mm})$ at varying intervals, and the paired-pulse ratio was calculated as the amplitude of the second (test) EJP divided by the amplitude of the first (conditioning) EJP. In control preparations, the test pulse was consistently larger than the conditioning pulse at all interstimulus intervals tested (Fig. $3 F$ ). In contrast, however, $\mathrm{dnl} 2^{70}$ mutants showed significantly less facilitation at all interstimulus intervals tested. This decrease in paired-pulse plasticity suggests an increase in transmitter release probability in $d n l 2^{70}$ mutants.

In addition to the differences in the amplitude of stimulusevoked transmitter release, we also observed differences in the kinetics of the EJPs and mEJPs in $d n l^{K O 70}$ mutants. Both the rise time and decay time of stimulus-evoked EJPs were significantly reduced in $d n l 2^{K O 70}$ mutants (Table 1, Fig. $3 G, H$ ). These reductions in rise time and decay time were observed in 0.8 and $0.2 \mathrm{~mm}$ calcium (Table 1, Fig. $3 G, H$ ). We also observed a reduction in the rise time of mEJPs, although we saw no significant difference in the decay time of mEJPs (data not shown). Together, these results suggest that $d n l 2$ is required at NMJs in Drosophila in which it plays an important role in regulating synaptic function.

\section{$d n l 2$ mutants have reduced numbers of synaptic boutons at the NMJ}

Studies in mammals have shown that loss of one or more neuroligins leads to defects in synaptic transmission without any significant defects in synaptic morphology (Varoqueaux et al., 2006; Chubykin et al., 2007). To determine whether the alterations in synaptic function observed in $d n l 2$ mutants are attributable to underlying morphological changes in the number or organization of synapses, we analyzed the overall pattern of innervation at the NMJ in both WT and $d n l 2$ mutants by immuno- 

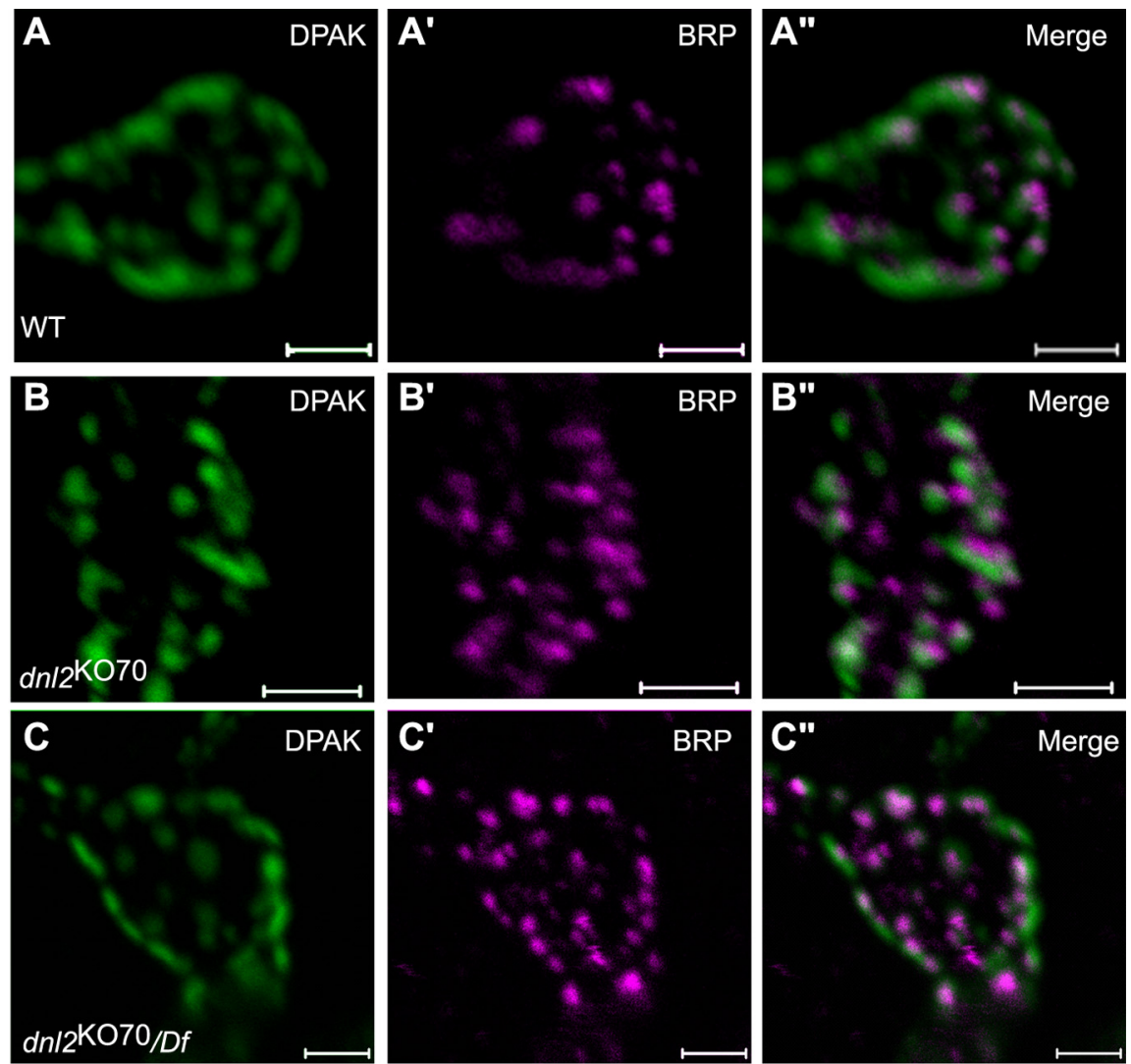

D
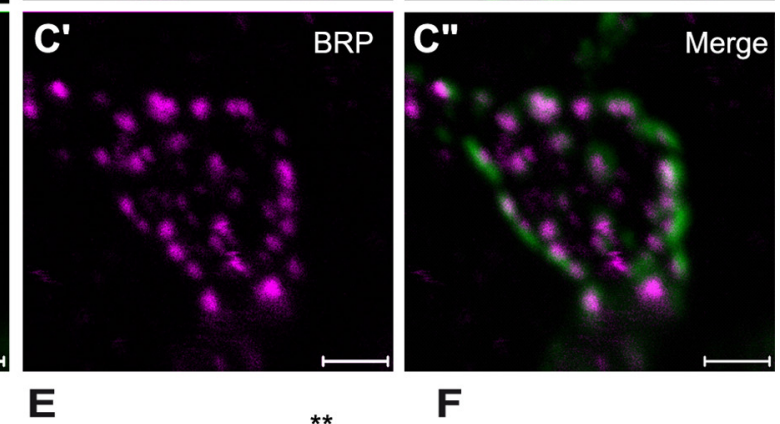

$\mathbf{F}$
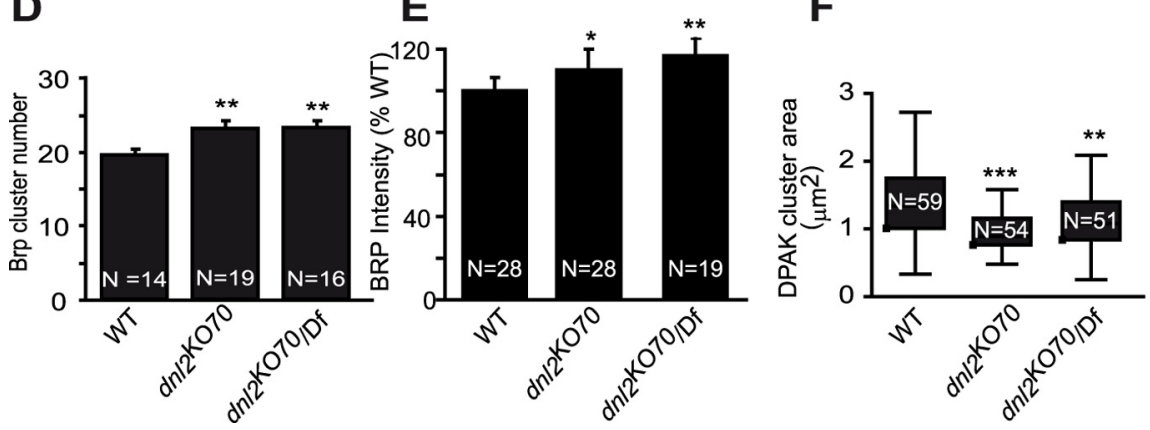

Figure 5. $d n / 2$ mutants have altered active zones and PSDs. $\boldsymbol{A}-\boldsymbol{C}^{\prime \prime}$, Confocal images of individual NMJ boutons costained with nc82 (anti-Brp) and anti-DPAK showing an increase in the number of BRP and DPAK staining clusters in $d n / 2$ mutants $\left(\boldsymbol{B}, d n / 2^{K 070}\right.$; $\left.\boldsymbol{C}, d n / 2^{K 070} / D f\right)$ and a reduction in the size of DPAK staining clusters in $d n / 2$ mutants compared with WT control. $\boldsymbol{D}$, The total number of DPAK clusters per bouton was increased in dn/2 mutants. $\boldsymbol{E}$, The average staining intensity of Brp spots was also increased in $d n / 2$ mutants. $\boldsymbol{F}$, Summary graph showing a small but significant decrease in the mean size of DPAK clusters compared with WT control. Box plots depict the 25 and $75 \%$ quartiles (top and bottom edges of the box) and the minimum and maximum values (bottom and top bars). ${ }^{*} p<0.05$ versus WT, ${ }^{* *} p<0.01$ versus WT, ${ }^{* * *} p<0.001$ versus WT, Mann-Whitney test. Scale bar, $1 \mu \mathrm{m}$.

formed in $d n l 2$ mutants and that the overall pattern of innervations was similar between WT and $d n l 2$ mutants (Fig. $4 A, B$ ), suggesting that $d n l 2$ is not absolutely required for synapse formation. Closer examination however, showed that the number of boutons was significantly reduced in the mutants compared with WT controls (Table 2, Fig. 4G). The decreased numbers of boutons was also associated with a significant decrease in the extent of synaptic arborization in $d n l 2$ mutants (Table 2, Fig. 4I). To confirm these results independently, we then used RNAi (Fire et al., 1998) to knockdown dnl2 expression. Specifically, we used the $24 \mathrm{~B}-\mathrm{Gal} 4$ driver, which is expressed in embryonic and larval muscle, to drive expression of a UAS- $d n l 2^{R N A i}$ construct and observed a significant reduction in bouton number and synaptic arborization (Table 2, Fig. 4D, G$I)$. To confirm that these deficits were attributable to a specific reduction in the level of Dnl2 protein, we performed rescue experiments whereby a WT UAS- $d n l 2$ transgene was expressed in the mutant muscle using either the 24B-Gal4 driver or the C57-Gal4 driver, which drives expression in all larval muscles, from mid first- to thirdinstar larval stages (Budnik et al., 1996; Packard et al., 2002). As shown in Figure $4 E-I$, both bouton number and synaptic arborization were fully restored to WT levels (Table 2). Together, the results suggest that, although $d n l 2$ is not absolutely required for synapse formation, it is required for proper proliferation and/or maturation of synaptic boutons during larval development.

\section{Increased active zone number and} reduced PSD size in $d n l 2$ mutants

Enhanced synaptic responses accompanied by a reduction in the number of synaptic boutons suggested to us that the properties within individual boutons were altered in $d n l 2$ mutants. To test this possibility, we examined presynaptic active zones (AZs) and the postsynaptic density. To assess presynaptic active zones, we used a

staining for HRP, a pan-neuronal marker widely used for assessing synapse morphology and the numbers of synaptic boutons. Specifically, we examined axon branching and bouton numbers at muscles $6 / 7$ and muscle 4 of abdominal segment 3 in third-instar larvae. Muscles 6/7 are innervated by two axons, the $\mathrm{MN6/7-Ib}$ and the MNSNb/d-Is, which give rise to type $\mathrm{Ib}$ and Is boutons, respectively (Hoang and Chiba, 2001). Muscle 4 is innervated by three different neurons, the MN4-Ib and the MNISN-Is, which give rise to type Ib and type Is boutons, respectively, and the MNISN-II, which gives rise to type II boutons (Hoang and Chiba, 2001). Only the type Ib boutons on muscle 4, however, were counted in this analysis, whereas all boutons on muscles 6/7 were counted. During initial examination of the NMJs in control and $d n l 2$ mutants, we found that synapses were monoclonal antibody against Brp, a synaptic marker known to specifically label the AZs at the NMJ synapse (Wucherpfennig et al., 2003). To assess postsynaptic organization, we examined the expression pattern of p21-activated protein kinase (PAK). The PAKs are a family of serine/threonine kinases that serve as targets for the small GTP-binding proteins $\mathrm{Cdc} 42$ and Rac and are important in the regulation of cytoskeleton organization (Bagrodia and Cerione, 1999). In Drosophila, DPAK has been shown to specifically localize in the PSD of the NMJ synapse (Wan et al., 2000).

In both WT and $d n l 2$ mutant larvae, Brp staining clusters were prominent at the NMJ (Fig. $5 A-C$ ), indicating that active zones could be formed in the mutants. Interestingly, the total number of Brp clusters per bouton was significantly increased in the mutants compared with WT controls (Table 2, Fig. 5D). The average 

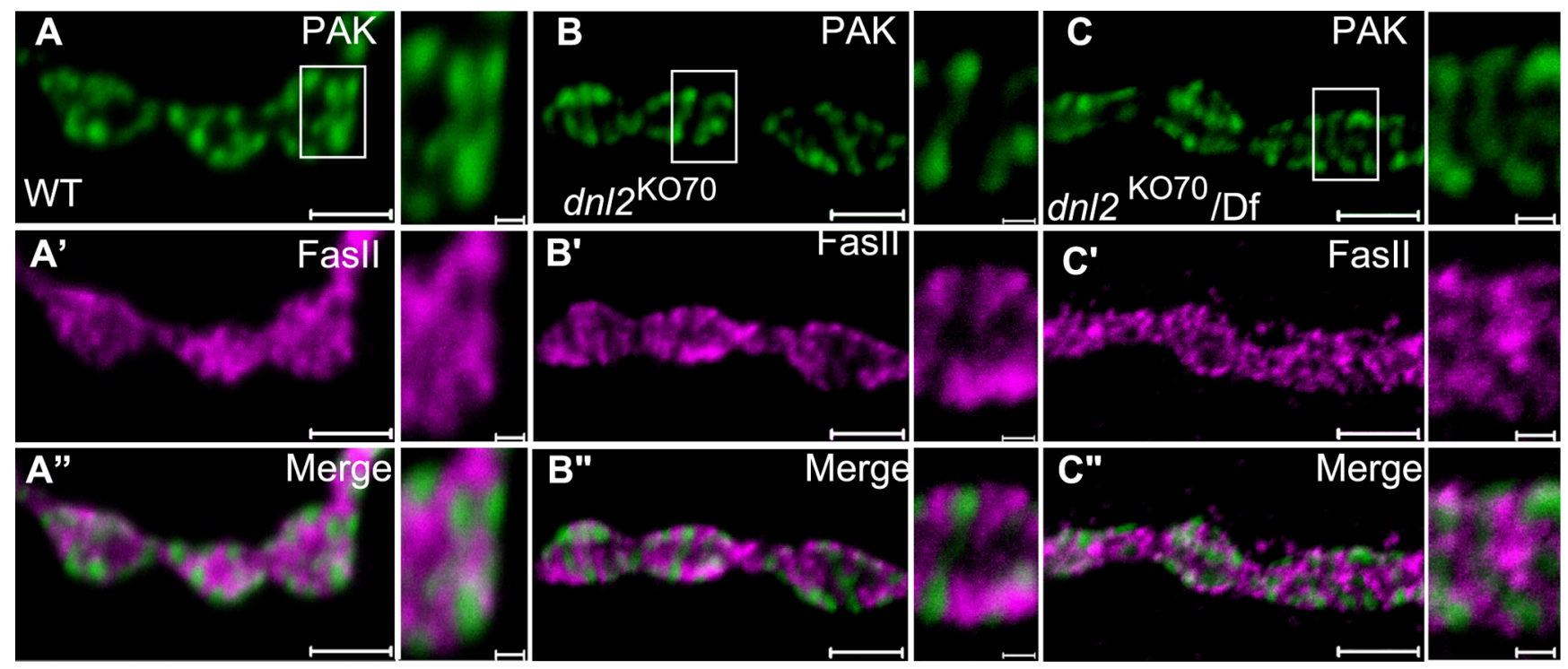

Figure 6. Organization of the postsynaptic density is normal in $d n / 2$ mutants. NMJ boutons were costained with anti-DPAK and anti-Fas II antibodies. In both the WT ( $\boldsymbol{A})$ and $d n / 2$ mutants ( $\boldsymbol{B}-\boldsymbol{C}$, DPAK clusters closely appose Fas II clusters. Although the number and size of DPAK clusters was altered in dn/2 mutants (Fig. 5), the DPAK clusters were still closely apposed to Fas II clusters in both $\mathrm{dn} / 2^{K 070}(\boldsymbol{B})$ and $d n 2^{K O 70} / \mathrm{Df}(\boldsymbol{C})$ mutants. Scale bar, $2 \mu \mathrm{m}$.

intensity of individual Brp clusters was also increased in $d n l 2$ mutants (Table 2, Fig. 5A-C,E).

In both WT and $d n l 2$ mutants, DPAK antibodies labeled well defined patches adjacent to the Fas II clusters along the surface of the bouton (Fig. 6A). In dnl2 null mutants, however, the total number of DPAK staining clusters per bouton was significantly higher compared with WT controls (Table 2, Fig. 6A-C). Interestingly, the mean size of individual DPAK staining clusters was significantly decreased (Table 2, Fig. 5F). However, the average intensity of staining in DPAK clusters was not altered in $d n l 2$ mutants (Table 2). The increased density of active zones and PSDs observed in $d n l 2$ mutants suggests a role for $d n l 2$ in bouton maturation/development.

\section{Ultrastructural abnormalities of NMJ synapses in dnl2 mutants}

We next examined synaptic ultrastructure using electron microscopy. In WT NMJ synapses, AZs are characterized by the presence of electron-dense T-shaped structures called "T-bars" in the presynaptic terminal (Atwood et al., 1993; Guan et al., 1996) (Fig. $7 A$, arrow). The electron-dense PSDs are in direct opposition to the T-bars and can be easily recognized and quantified. In $d n l 2$ mutants, individual T-bars appeared to be normal in shape, but the total number of these structures per bouton was significantly increased (Table 2, Fig. $7 A, B, C, E$ ). The increase in the number of active zones was rescued by expressing a WT UAS- $d n l 2$ transgene in the mutant muscle using the 24B-Gal4 driver (Table 2, Fig. $7 D, E)$. This result was consistent with the immunostaining data using the AZ marker Brp. We also observed defects in postsynaptic organization. Specifically, the length of PSDs was significantly reduced in $d n l 2$ mutants (Table 2, Fig. $7 F$ ). The thickness of the SSR structures was also reduced in $d n l 2$ mutants (Table 2, Fig. $7 G$ ). Importantly, the changes in both PSD length and SSR thickness could be rescued by expression of a wild-type $d n l 2$ transgene in the muscle (Table 2, Fig. $7 D, F, G$ ).

$d n l 2$ mutants have reduced postsynaptic glutamate receptors in single boutons

To determine whether the defects in PSD size and number affected postsynaptic receptor density, we examined glutamate re- ceptor (DGluR) expression at individual boutons. Previous work has identified a total of five DGluR subunits (Fig. 8A) within Drosophila muscles (GluRIIA, IIB, III, IID, and IIE) (Schuster et al., 1991; Petersen et al., 1997; DiAntonio et al., 1999; Marrus and DiAntonio, 2004; Marrus et al., 2004; Featherstone et al., 2005; Qin et al., 2005) from which two distinct receptor complexes containing either GluRIIA or GluRIIB plus the other subunits are assembled (Fig. 8A). GluRIIA- or GluRIIB-containing receptor complexes can coexist within individual NMJ synapses; furthermore, recent work by Chen et al. (2010) showed a shift in the ratio of GluRIIA to GluRIIB receptor complexes in favor of GluRIIA complexes in embryonic neurexin mutants. Because GluRIII is a common subunit for both receptor complex types, we used the expression level of GluRIII to assess the total amount of synaptic GluRs. As shown in Figure 8, $B$ and $E$, the level of synaptic GluRIII was dramatically reduced in $d n l 2$ mutants compared with the WT control (Table 2, Fig. 8E). Similarly, GluRIIB staining was also significantly reduced in $d n l 2$ mutants (Table 2 , Fig. $8 D, G$ ). Interestingly, despite an overall decrease in the total density of GluRs, we observed a small but significant increase in the level of GluRIIA in $d n l 2$ mutants (Table 2, Fig. 8C,F). These defects in GluR density were only partially rescued by postsynaptic expression of a full-length $d n l 2$ transgene (Table 2, Fig. $8 E-G$ ). These results indicate that $d n l 2$ plays a role in targeting GluRs to the PSD.

\section{Interaction between $d n l 2$ and neurexin}

Studies in mammals suggest that Neuroligins function as transsynaptic receptors for Neurexins and that binding of Neuroligin to Neurexin is important for many of its functions (Südhof, 2008). To determine whether the observed defects in $d n l 2 \mathrm{mu}-$ tants are attributable to an interaction with neurexin, we first sought to determine whether $d n l 2$ can bind to $d n r x$. Using lysates from adult heads, we found that anti-Dnrx antibodies were able to immunoprecipitate Dnl2, and anti-Dnl2 antibodies were able to immunoprecipitate Dnrx (Fig. 9A) demonstrating that Dnl2 and Dnrx form a complex in vivo.

We next asked whether the observed defects in NMJ morphology are affected by loss of $d n r x$. Homozygous $d n l 2^{K O 70}$ or $d n r x^{\Delta 83}$ (Zeng et al., 2007) flies are viable and fertile. In contrast, homozy- 

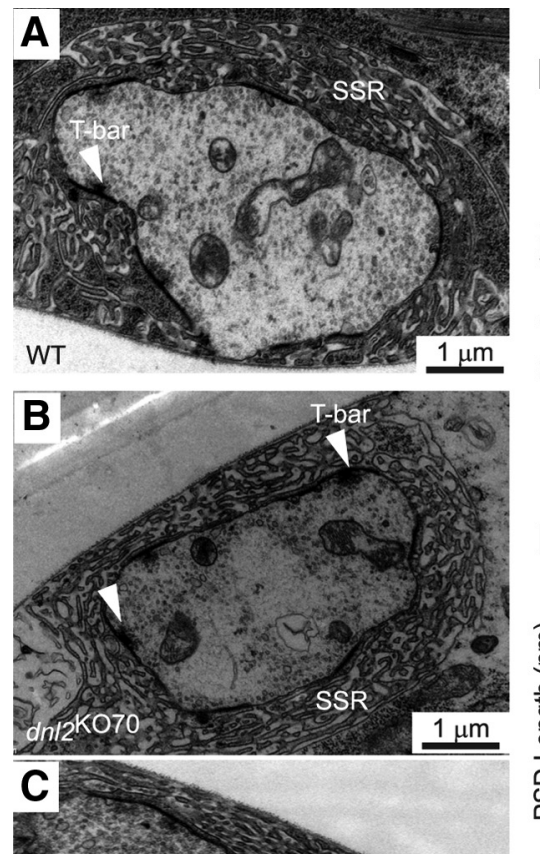

$\mathbf{E}$
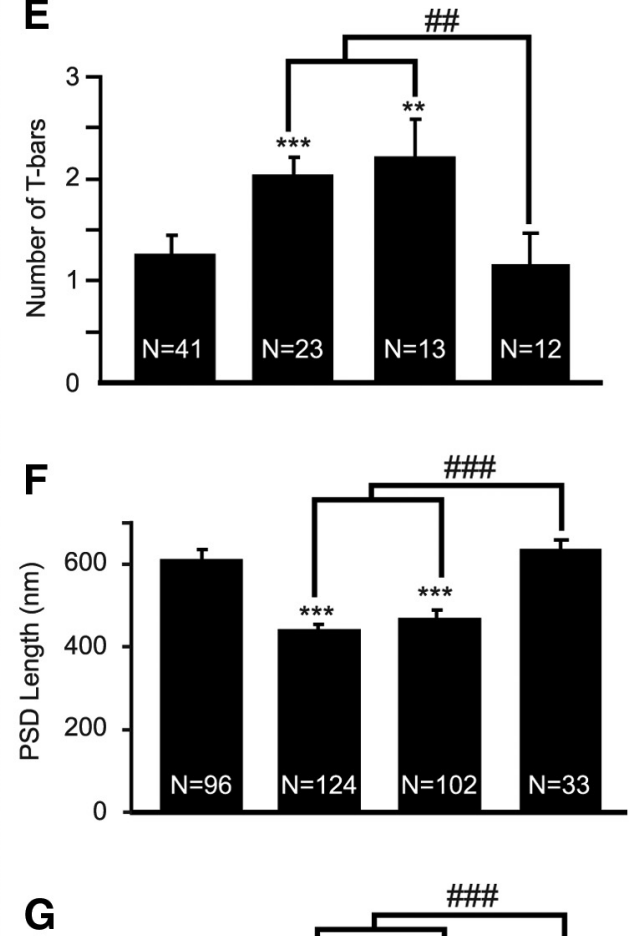

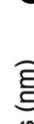

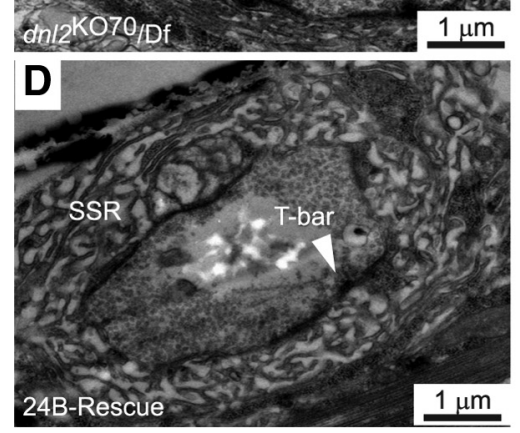

Figure 7. Ultrastructural analysis of type I synaptic bouton in $d n / 2$ mutants. $A-D$, Representative transmission electron microscope micrographs showing low-magnification views of synaptic boutons, the presynaptic active zones (arrowheads), and postsynaptic SSR. $\boldsymbol{E}$, Morphometric analysis of reconstructed synaptic boutons showed a significant increase in the number of T-bars per bouton in $d n / 2$ mutants that was rescued by muscle specific expression of a UAS-dn/2 CDNA with 24B-Gal4. $\boldsymbol{F}$, The length of PSDs beneath presynaptic T-bars was reduced in $d n 12$ mutants. This was also rescued by muscle expression of UAS-dn/2 with 24B-Gal4. G, The SSR was thinner in dn/2 mutants compared with WT controls or 24B-rescue flies. All images and analyses were derived from type lb boutons on muscles 6/7. ${ }^{* *} p<0.01$ versus WT, ${ }^{* * *} p<0.001$ versus WT, ${ }^{* \#} p<0.01$ versus $\mathrm{dn} / 2^{K 070}$, $\# \#<0.001$ versus $d n / 2^{K 070}$, Mann-Whitney test.

gous $d n l 2 ; d n r x^{\Delta 83}$ double-mutant animals die during the secondinstar larval stage. Because of the early lethality of the $d n l 2 ; d n r x^{\Delta 83}$ double mutants, we first examined synaptic morphology in early second-instar larvae. Even at this earlier developmental stage, $d n l 2^{K O 70}$ mutants showed a significant reduction in bouton numbers compared with WT (Table 3, Fig. 9B, C,F). We also observed a similar reduction in bouton numbers in $d n r x^{\Delta 83}$ mutants (Table 3, Fig. 9D,F). These results are consistent with several recent observations showing similar morphological defects in independently generated $d n r x$ and $d n l 1$ mutants (Li et al., 2007b; Banovic et al., 2010; Chen et al., 2010). In contrast to the results of Banovic et al. (2010), however, when we examined $d n l 2 ; d n r x^{\Delta 83}$ double mutants, we saw an even more severe defect in NMJ morphology (Fig. 9E,F). Consistent with the defects in synaptic morphology, $d n l 2 ; d n r x^{\Delta 83}$ double mutants also showed more severe defects in locomotion than either $d n l 2$ or $d n r x$ mutants alone (Table 3, Fig. 9G).

We also generated flies that were homozygous mutant for $d n l 2$ and lacked one copy of $d n r x\left(d n l 2^{K O 70} / d n l 2^{K O 70}\right.$; $\left.d n r x^{\Delta 83} /+\right)$ as well as flies that were homozygous mutant for $d n r x$ and lacked one copy of $d n l 2\left(d n l 2^{K O 70} /+\right.$; $\left.d n r x^{\Delta 83 /} d n r x^{\Delta 83}\right)$. These hemizygous $d n l 2 ; d n r x$ mutants were viable, allowing us to analyze NMJ morphology in third-instar larvae. When we examined the NMJ morphology of these mutants, we noted that $d n l 2$ mutants with only one copy of $d n r x$ showed more severe defects in NMJ morphology at both muscles 6/7 (Table 3, Fig. 9H-M) and muscle 4 (Table 3, Fig. $9 G^{\prime}-M^{\prime}$ ) than $d n l 2$ mutants alone. Similarly, dnrx mutants with only one copy of $d n l 2$ also showed more severe defects in NMJ morphology at both muscles 6/7 (Table 3, Fig. $9 M$ ) and muscle 4 (Table 3, Fig. $\left.9 B^{\prime}-G^{\prime}\right)$ than $d n r x$ mutants alone. Together, these results suggest that, although $d n r x$ and $d n l 2$ bind and are both required for synapse formation and function, dnrx must also interact with additional genes/pathways.

\section{Discussion}

Neurexins and Neuroligins are highly conserved cell adhesion molecules that form an asymmetric, trans-synaptic complex required for synapse formation (Südhof, 2008). In the present study, we have examined a homolog of neuroligin $(d n l 2)$ in Drosophila expressed at NMJ synapses and in the CNS. We show that $d n l 2$ null mutants are viable and exhibit numerous defects in synaptic morphology and function. Presynaptically, we observed a reduction in axonal branching and fewer synaptic boutons, although the number of active zones per bouton was increased. Postsynaptically, dnl2 mutants exhibited a decrease in GluR density and a shift in the ratio of GluRIIA to GluRIIB receptor complexes in favor of GluRIIA complexes. dnl2 mutants also showed a decrease in complexity of the subsynaptic reticulum. Both presynaptic and postsynaptic defects observed in $d n l 2 \mathrm{mu}-$ tants could be recapitulated by knockdown of $d n l 2$ in muscle, and, more importantly, the defects could be rescued by postsynaptic expression of a wild-type $d n l 2$ transgene. Functionally, $d n l 2$ mutants showed an increase in transmitter release and a decrease in pairedpulse plasticity indicative of an increase in transmitter release probability. It is also possible that changes in the active (voltage-gated) properties of the postsynaptic membrane may contribute to the increased amplitude of EJPs. Indeed, the changes in the kinetics of EJPs with little or no change in the kinetics of mEJPs may be indicative of altered membrane conductance. Together, these data indicate that, although $d n l 2$ is not absolutely required for synaptogenesis, it does play an important role in the postsynaptic cell in synapse maturation and function. 

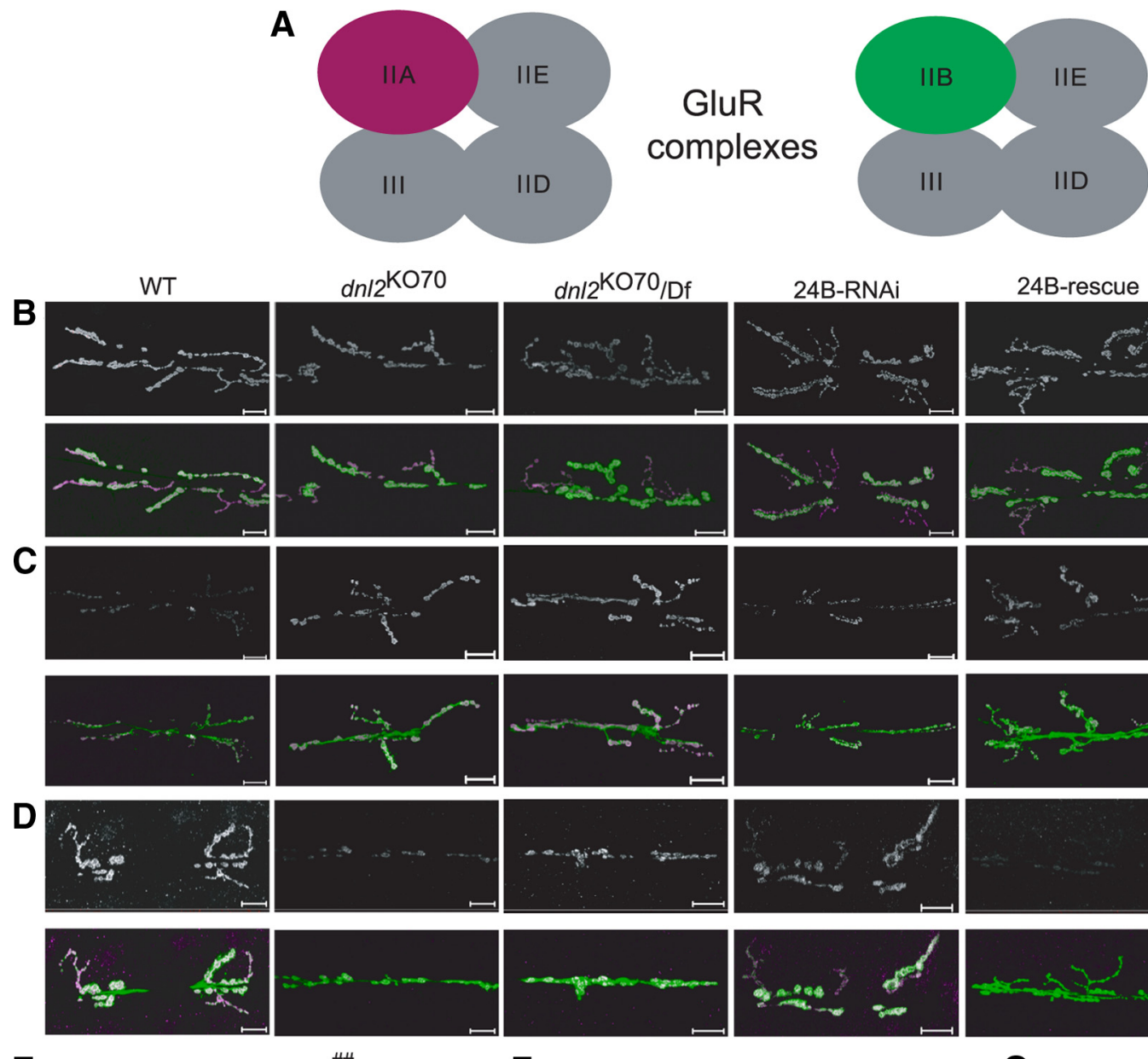

24B-rescue

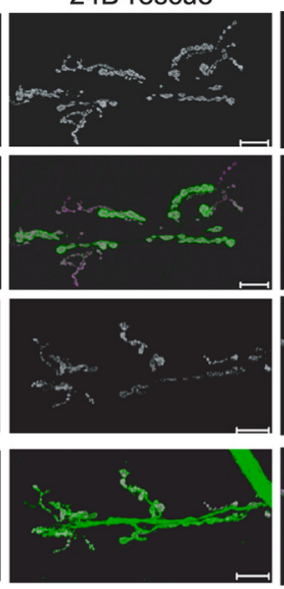

C57-rescue

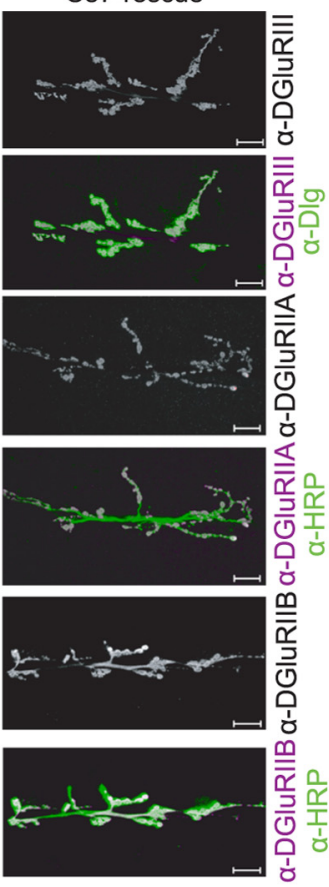

E
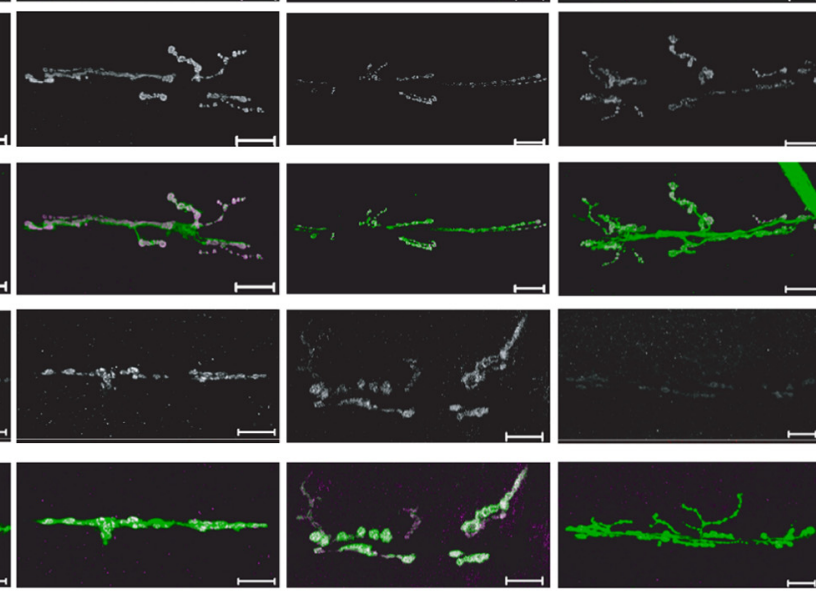

$F$
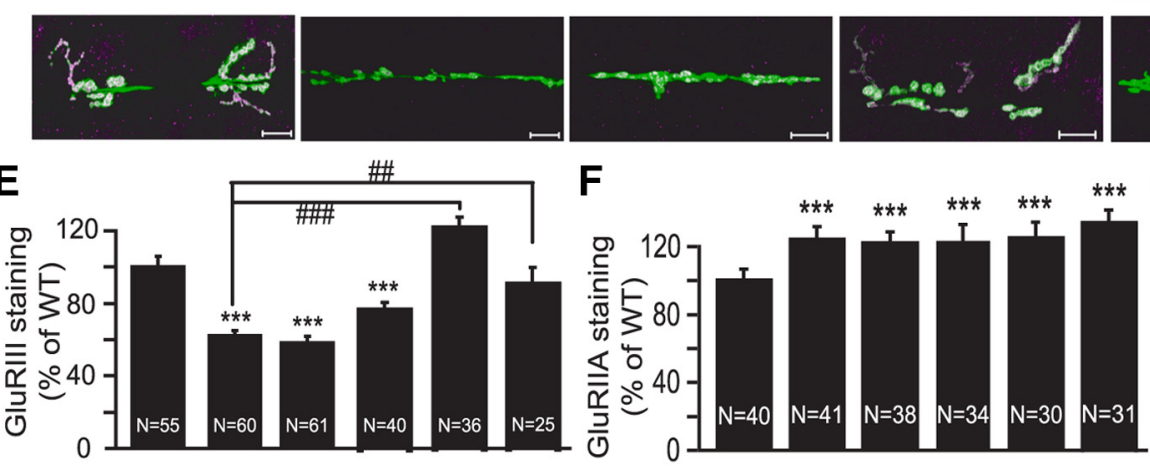

G

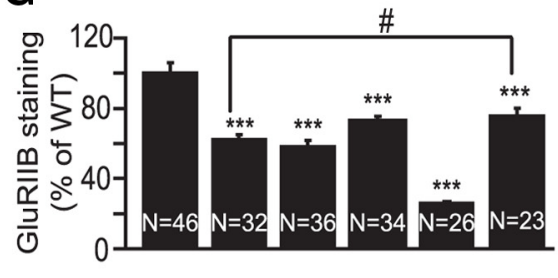

E'

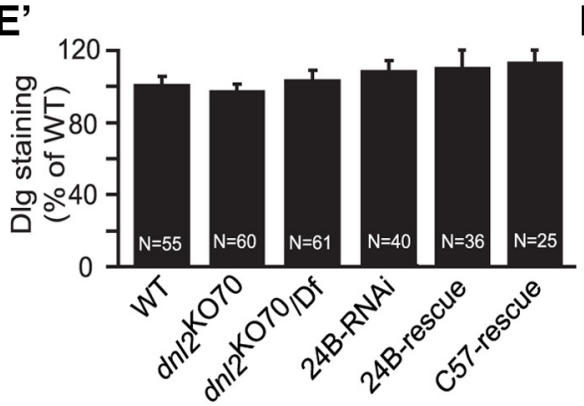

F'

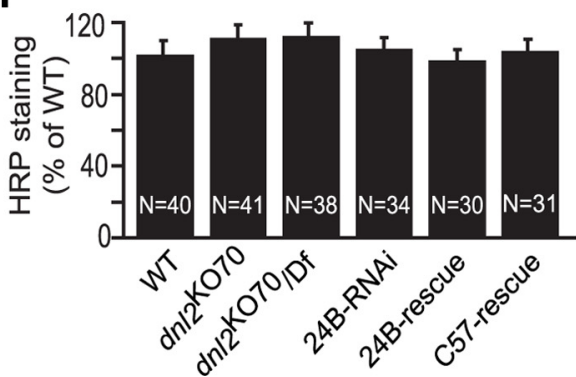

G'

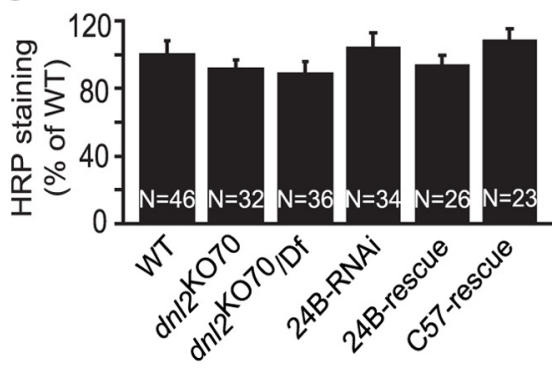

Figure 8. dn/2 mutants exhibit changes in DGluRs abundance at the NMJ. $A$, Subunit composition of two major glutamate receptor complexes at the NMJ: each receptor complex contains GluRIIIA or GluRIIB alongside one of each of the other three subunits. $\boldsymbol{B}-\boldsymbol{D}$, Representative NMJs from WT, dn/2 null mutants (homozygous $d n / 2^{\text {KO }}{ }^{20}$ and $d n / 2^{\text {KO70 } / D f), ~ d n / 2--R N A i ~ t r a n s g e n i c ~ f l i e s ~(U A S-d n / 2 ~}$ RNAi driven in muscles by 24B-Gal4), and two rescue strains expressing a UAS-dn/2 CDNA in the dn/2 mutant using either 24B-Gal4 or C57-Gal4 (both muscle drivers). NMJs were costained with anti-DGluRIII and anti-Dlg $(\boldsymbol{B})$, anti-GluRIIA and anti-HRP $(\boldsymbol{C})$, or anti-GluRIIB and anti-HRP $(\boldsymbol{D})$. $\boldsymbol{E}-\boldsymbol{G}^{\prime}$, Summaries of normalized fluorescence intensities for DGluRIII $(\boldsymbol{E})$, DGluRIIA $(\boldsymbol{F})$, and DGluRIIIB (G) compared with normalized fluorescence intensities for $\operatorname{Dlg}\left(\boldsymbol{E}^{\prime}\right)$ or $\operatorname{HRP}\left(\boldsymbol{F}^{\prime}, \boldsymbol{G}^{\prime}\right)$ in the same boutons. Data are expressed as a percentage of WT fluorescence intensity. Our results show a significant reduction in the levels of DGluRIII $(\boldsymbol{E})$ and GluRIIB $(\boldsymbol{G})$, without any significant change Dlg $\left(\boldsymbol{E}^{\prime}\right)$ or HRP $\left(\boldsymbol{G}^{\prime}\right)$ in both dn/2 null and 24B-dn/2 RNAi transgenic flies compared with WT control. We also saw an increase in GluRIIA $(\boldsymbol{F})$ in dn/2 mutants and 24B-dn/2 RNAi flies without any change in HRP $\left(\boldsymbol{F}^{\prime}\right)$. Expression of a UAS-dn/2 transgene in the muscles was able to partially rescue the defects in GluR expression. Specifically, the decrease in GluRIII expression was rescued by expression of the UAS-dn/2 in the muscle with either 24B-Gal4 or C57-Gal4. The decrease in GluRIIB expression was partially rescued using the (57-Gal4 driver but not using the 24B-Gal4 driver. The increase in GluRIIA expression, however, was not rescued with either of the muscle drivers. ${ }^{* * *} p<0.001$ versus WT, ${ }^{\#} p<0.05$ versus $d n / 2^{K 070, ~}{ }^{\# \#} p<0.01$ versus $d n / 2^{K 070}$, and ${ }^{\# \# \#} p<0.001$, Mann-Whitney test. 
A
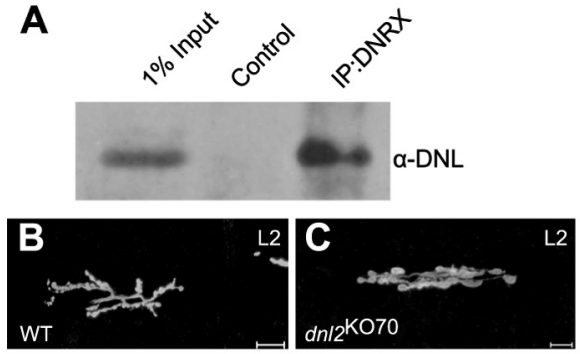

$\mathbf{F}$
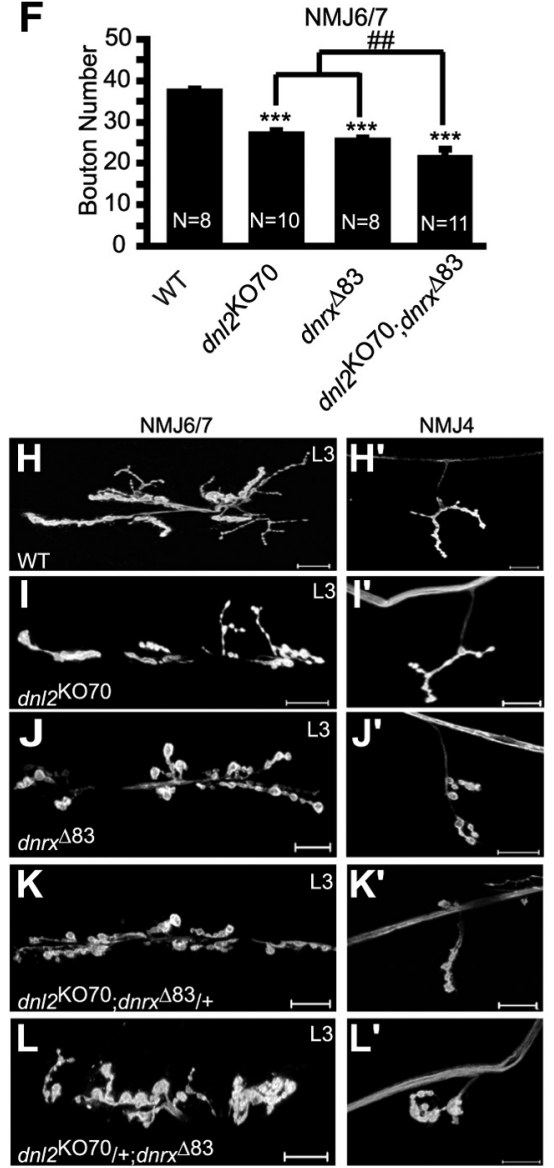
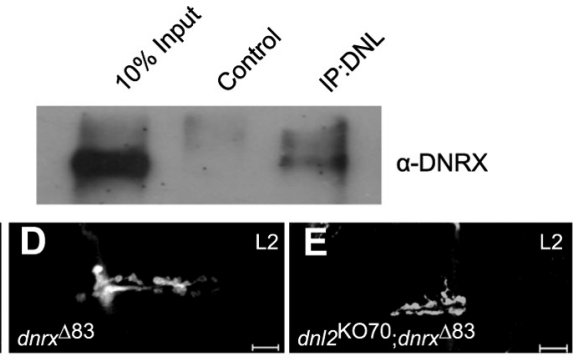

G

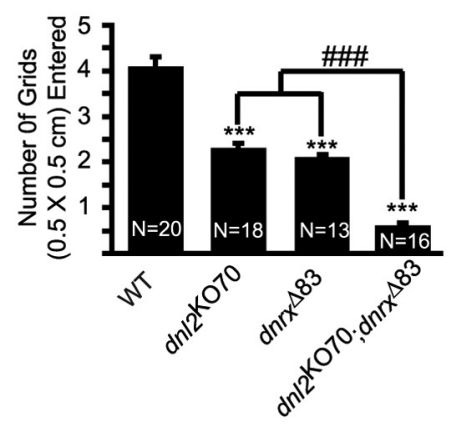

M

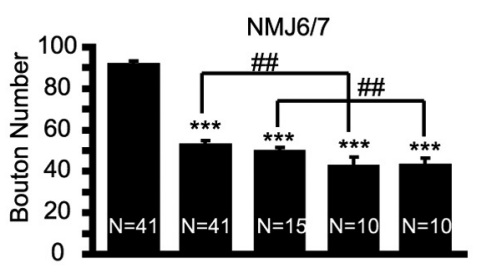

M'

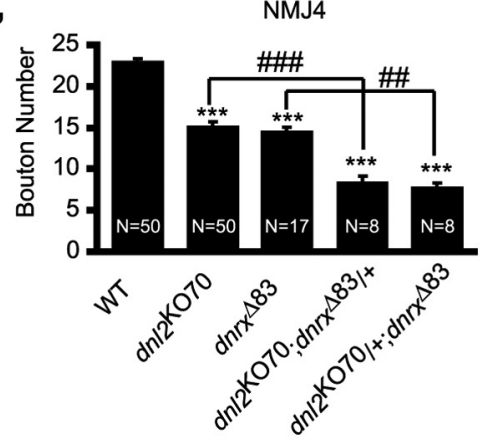

Figure 9. Genetic interaction between $d n / 2$ and $d n r x$ in NMJ development and larval locomotion. $\boldsymbol{A}$, Fly head homogenates from adult WT flies were immunoprecipitated (IP) with anti-DNRX monoclonal antibody and control monoclonal antibody lgG using protein A/G Sepharose beads. One percent of input homogenate and coimmunoprecipitates were analyzed by immunoblotting using anti-Dnl2 antibody. Anti-Dnrx antibody precipitated the DnI2 from the lysates, whereas the control lgG did not. Meanwhile, anti-Dnl2 antibody precipitated the Dnrx from the lysates in which the control lgG did not. $\boldsymbol{B}-\boldsymbol{E}$, Representative NMJs on muscles 6/7 of second-instar larvae in WT, $d n / 2^{K 070}, d n r x^{\Delta 83}$ (Zeng et al., 2007), and $d n / 2 ; d n r x$ double mutants stained with anti-HRP and anti-Dlg. F, G, Quantification of NMJ morphology and locomotor activity in early second-instar larvae. The $d n / 2 ; d n r x$ double-mutant larvae displayed more severe defects in bouton numbers and locomotor activity than either the $d n / 2$ or $d n r x$ mutants alone. ${ }^{* * *} p<0.001$ versus WT, Mann-Whitney test; ${ }^{\# \#} p<0.01$ versus $d n / 2^{K 070}$ and $d n r x^{\Delta 83}$, \#\#\# $p<0.001$ versus $d n / 2^{K 070}$ and $d n r x^{\Delta 83}$, Kruskal-Wallis test. $\boldsymbol{H}-\boldsymbol{M}$, NMJ morphology at muscle $6 / 7(\boldsymbol{H}-\boldsymbol{L})$ and muscle $4\left(\boldsymbol{H}^{\prime}-\boldsymbol{L}^{\prime}\right)$ in abdominal segment 3 of third-instar larvae labeled with anti-HRP and anti-Dlg. Compared with wild-type $\left(\boldsymbol{H}, \boldsymbol{H}^{\prime}\right), d n / 2\left(d n / 2^{K 070} ; \boldsymbol{I}, \boldsymbol{I}^{\prime}\right)$, and $d n r x\left(d n r x^{\Delta 83} ; \boldsymbol{J}, \boldsymbol{J}^{\prime}\right)$ null mutants show less NMJ expansion and fewer boutons. Loss of one copy of dnrx in $d n / 2^{K 070}$ larvae $\left(\boldsymbol{K}, \boldsymbol{K}^{\prime}\right)$ or loss of one copy of $d n / 2$ in dnrx ${ }^{\Delta 83}$ homozygous larvae $\left(\boldsymbol{L}, \boldsymbol{L}^{\prime}\right)$ led to even greater reductions in bouton numbers. Scale bars, 20 $\mu \mathrm{m} . \boldsymbol{M}, \boldsymbol{M}^{\prime}$, Quantification of total bouton numbers at NMJs from 6/7 $(\boldsymbol{M})$ and type lb bouton number at NMJ4 $\left(\boldsymbol{M}^{\prime}\right) .{ }^{* * *} p<0.001$ versus WT, ${ }^{\# \#} p<0.01$ versus $d n / 2^{K 070}$ and $d n r x^{\Delta 83, \# \# p} p<0.001$ versus $d n / 2^{K 070}$ Kruskal-Wallis test.

$d n l 2$ regulates presynaptic and postsynaptic development $d n l 2$ mutants showed several defects in postsynaptic architecture. In vertebrates, neuroligins are thought to regulate postsynaptic organization via a direct interaction with PSD-95 (Irie et al., 1997). In Drosophila, the homolog of PSD-95, $d l g$, has been shown to be required for several aspects of postsynaptic organi- zation (Lahey et al., 1994; Budnik et al., 1996; Guan et al., 1996; Chen and Featherstone, 2005). Furthermore, the defects in postsynaptic architecture in $d n l 2 \mathrm{mu}-$ tants are reminiscent of a defect in $d l g$ function. Loss of $d n l 2$ does not appear to prevent or impair clustering of $d l g$ at postsynaptic sites because the overall $d l g$ levels was not different in $d n l 2$ mutants. Rather, $d n l 2$ may be required for proper dlg signaling.

dnl2 mutants also showed several defects in presynaptic architecture mediated by trans-synaptic interactions. The most likely candidate for a trans-synaptic signaling partner is neurexin. Both $d n l 2$ and $d n r x$ null flies are viable but display significant reductions in the number of synaptic boutons. We observed strong colocalization of $d n l 2$ and $d n r x$ in the CNS and the NMJ and were able to detect a complex between the two proteins in vivo. dnl2; $d n r x$ double mutants, however, are lethal and display more severe phenotypes than those observed in single mutants, implying that $d n l 2$ and $d n r x$ may interact with additional partners during synaptic development. For example, dnrx can also interact with other neuroligins in Drosophila to mediate synaptic development. Banovic et.al. (2010) found that loss of $d n r x$ did not enhance the morphological defects in dnl1 mutants, suggesting that both genes function within a common pathway. Furthermore, a point mutation in $d n l l$ that is predicted to abolish binding to dnrx suppressed the phenotype associated with overexpression of $d n l 1$ (Banovic et al., 2010). There are also two other predicted homologs of neuroligin in flies, but the function of these genes remains to be determined. Neuroligins and neurexins may also form additional complexes with other proteins involved in synaptogenesis. A recent study found that neuroligin was able to induce increases in synaptic density independently of neurexin binding (Ko et al., 2009b). Similarly, two other recent studies showed that leucine-rich repeat transmembrane proteins can induce presynaptic differentiation when bound to neurexin (de Wit et al., 2009; Ko et al., 2009a), providing a novel trans-synaptic neurexin-dependent mechanism for development of presynaptic specializations.

If the presynaptic defects observed in $d n l 2$ mutants are not mediated via an interaction with neurexin, the question remains, how does $d n l 2$ affect presynaptic morphology? One possibility is that these changes occur indirectly. The level of postsynaptic GluRIIA expression is correlated with changes in the number of presynaptic T-bars (Sigrist et al., 2002). It is possible that GluRIIA expression is regulated in part by $d n l 2$, and increased GluRIIA expression in 
Table 3. NMJ morphology in dn/2;dnrx double mutants

\begin{tabular}{|c|c|c|c|c|c|c|}
\hline & $w^{1118}$ & $d n / 2^{K 070}$ & $d n r x^{\Delta 83}$ & $d n / 2^{K 070} ; d n r x^{\Delta 83}$ & $d n / 2^{K 070}, d n r x^{\Delta 83} /+$ & $d n 12^{K 070} /+; d n r x^{\Delta 83}$ \\
\hline \multicolumn{7}{|l|}{ Second-instar larvae } \\
\hline NMJ boutons & $37.6 \pm 0.9$ & $26.8 \pm 1.3$ & $25 \pm 0.7$ & $21.1 \pm 1.4$ & ND & ND \\
\hline Locomotion index & $4 \pm 0.25$ & $2.2 \pm 0.2$ & $2 \pm 0.1$ & $0.6 \pm 0.1$ & ND & ND \\
\hline \multicolumn{7}{|l|}{ Third-instar larvae } \\
\hline m6/7 boutons & $91.4 \pm 1.8$ & $52.5 \pm 2.4$ & $48.8 \pm 1.9$ & Lethal & $39.8 \pm 1.9$ & $39.4 \pm 1.8$ \\
\hline $\mathrm{m} 4$ boutons & $22 \pm 0.6$ & $14.9 \pm 0.5$ & $14.5 \pm 0.5$ & Lethal & $9.9 \pm 0.8$ & $9.6 \pm 1.2$ \\
\hline
\end{tabular}

In Second-instar larvae: NMJ boutons, total number of $\mathrm{lb}$ and $\mathrm{ls}$ boutons on muscles 6 and 7 of abdominal segment 3; Locomotion index, number of grids $(0.5 \times 0.5 \mathrm{~cm})$ entered. In Third-instar larvae: $\mathrm{m} 6 / 7$ boutons, number of $\mathrm{lb}$ and $\mathrm{ls}$ boutons on muscles 6 and 7 of abdominal segment $3 ; \mathrm{m} 4$ boutons, number of $\mathrm{lb}$ boutons on muscle 4 of abdominal segment 3 . ND, No data for that genotype.

dnl2 mutants is responsible for the increased density of T-bars. Normally, the density of T-bars in individual boutons is held constant via homeostatic changes in the expression of the cell adhesion molecule Fas II (Schuster et al., 1996a,b; Meinertzhagen et al., 1998; Sigrist et al., 2002). In the present study, however, we observed an increase in the number of T-bars per bouton. Moreover, we saw a decrease in the total number of boutons rather than an increase as might be expected based on previous studies. Because the addition of synaptic boutons during NMJ growth requires the downregulation of Fas II expression, the uncoupling between T-bar numbers and bouton expansion in $d n l 2$ mutants may suggest that $d n l 2$ is involved in the GluRIIAmediated downregulation of Fas II.

\section{$d n l 1$ and $d n l 2$ have independent but overlapping functions at the NMJ}

The Drosophila genome is predicted to have four neuroligin homologs and a single neurexin homolog. Whether all four Drosophila neuroligins are required for synapse development is presently unknown. To date, the only other neuroligin that has been studied in Drosophila is dnl1 (Banovic et al., 2010). Neither $d n l 1$ nor $d n l 2$ are required for synaptogenesis, yet both genes play a role in the development/maturation of the NMJ, suggesting that the two genes may be functionally redundant. Both proteins are expressed at the NMJ in wild-type animals, and null mutations in either gene lead to significantly reduced bouton numbers, defects in GluR organization, and alterations in the complexity of the subsynaptic reticulum (Banovic et al., 2010).

Despite these similarities, however, there are also a number of differences between $d n l 1$ and $d n l 2$ null mutants. First, $d n l 2$ is expressed in both the CNS and muscles, whereas $d n l 1$ is only expressed in muscle. Second, $d n l 1$ mutants showed a complete loss of GluR expression in $\sim 10 \%$ of boutons (Banovic et al., 2010), whereas $d n l 2$ showed a uniform decrease in total GluR expression in all boutons and an increased abundance of GluRIIA receptor complexes at the expense of GluRIIB complexes. Third, dnll mutants showed a decrease in transmitter release (Banovic et al., 2010), whereas $d n l 2$ mutants showed an increase in transmitter release. Although both mutants showed a decrease in bouton number, $d n l 2$ mutants also showed a significant increase in the number of active zones. As such, the differences in the amplitude of transmitter release observed in $d n l 1$ and $d n l 2$ mutants likely reflect the different presynaptic morphologies of the two mutants.

Finally, there were differences in the interaction between the two neuroligin homologs and neurexin $(d n r x)$. $d n l 1$ shows very little colocalization with $d n r x$ and none outside the NMJ (Banovic et al., 2010). In contrast, $d n l 2$ showed a much stronger colocalization at the NMJ and also shows strong colocalization within the CNS. Furthermore, $d n l 2$ forms a complex with $d n r x$ in vivo, although it remains to be shown whether the same is true for $d n l 1$ (Banovic et al., 2010). $d n l 2 ; d n r x$ double mutants were lethal and showed more severe defects in bouton morphology than either $d n l 2$ or $d n r x$ mutants alone, whereas $d n l 1 ; d n r x$ mutants were viable and did not show any exacerbation of the morphological defects (Banovic et al., 2010). Together, these results suggest that the interactions between $d n l 1$ or $d n l 2$ and $d n r x$ serve different functions at the NMJ.

Because mutations in $d n r x$ or either of the two $d n l$ genes studied thus far all give rise to a reduction in the number of synaptic boutons, it seems likely that bouton number is regulated via an interaction between $d n r x$ and $d n l 1$ and/or $d n l 2$. It is also apparent, however, that these three genes perform functions independently of each other, such that single mutants have similar yet distinct synaptic phenotypes. Banovic et al. (2010) concluded that $d n r x$ promotes but is not necessary for $d n l 1$ function. The results of the present study, however, showing exaggerated synaptic phenotypes and lethality in $d n l 2$; $d n r x$ double mutants may suggest some redundancy between the functions of $d n r x$ and $d n l 2$. Consistent with this model, a recent publication showed that $d n r x$ is expressed both presynaptically and postsynaptically in embryonic NMJs (Chen et al., 2010). Furthermore, postsynaptic $d n r x$ appears to specifically promote GluRIIA receptor complexes (Chen et al., 2010). This raises an interesting possibility of a cis-interaction between $d n l 2$ and $d n r x$ in addition to transsynaptic interactions, although additional work will be required to assess whether cis-interactions occur, and if so, what role they play.

Together, the results of the present study combined with the results of Banovic et al. (2010) suggest that neither $d n l 1$ nor $d n l 2$ are absolutely required for synaptogenesis, but both genes play an essential role in synaptic development. Additional studies will be required to determine the function of other neuroligin genes in Drosophila and to determine whether these genes have functionally redundant roles in synapse development and function.

\section{References}

Ashley J, Packard M, Ataman B, Budnik V (2005) Fasciclin II signals new synapse formation through amyloid precursor protein and the scaffolding protein dX11/Mint. J Neurosci 25:5943-5955.

Atwood HL, Govind CK, Wu CF (1993) Differential ultrastructure of synaptic terminals on ventral longitudinal abdominal muscles in Drosophila larvae. J Neurobiol 24:1008-1024.

Bagrodia S, Cerione RA (1999) Pak to the future. Trends Cell Biol 9:350-355.

Banovic D, Khorramshahi O, Owald D, Wichmann C, Riedt T, Fouquet W, Tian R, Sigrist SJ, Aberle H (2010) Drosophila Neuroligin 1 promotes growth and postsynaptic differentiation at glutamatergic neuromuscular junctions. Neuron 66:724-738.

Bolliger MF, Frei K, Winterhalter KH, Gloor SM (2001) Identification of a novel neuroligin in humans which binds to PSD-95 and has a widespread expression. Biochem J 356:581-588.

Budnik V, Koh YH, Guan B, Hartmann B, Hough C, Woods D, Gorczyca M (1996) Regulation of synapse structure and function by the Drosophila tumor suppressor gene dlg. Neuron 17:627-640.

Chen K, Featherstone DE (2005) Discs-large (DLG) is clustered by presyn- 
aptic innervation and regulates postsynaptic glutamate receptor subunit composition in Drosophila. BMC Biol 3:1.

Chen K, Gracheva EO, Yu SC, Sheng Q, Richmond J, Featherstone DE (2010) Neurexin in embryonic Drosophila neuromuscular junctions. PLoS ONE 5:e11115.

Chih B, Engelman H, Scheiffele P (2005) Control of excitatory and inhibitory synapse formation by neuroligins. Science 307:1324-1328.

Chubykin AA, Atasoy D, Etherton MR, Brose N, Kavalali ET, Gibson JR, Südhof TC (2007) Activity-dependent validation of excitatory versus inhibitory synapses by neuroligin-1 versus neuroligin-2. Neuron 54:919-931.

Comoletti D, Flynn R, Jennings LL, Chubykin A, Matsumura T, Hasegawa H, Südhof TC, Taylor P (2003) Characterization of the interaction of a recombinant soluble neuroligin-1 with neurexin-1beta. J Biol Chem 278:50497-50505.

Craig AM, Graf ER, Linhoff MW (2006) How to build a central synapse: clues from cell culture. Trends Neurosci 29:8-20.

de Wit J, Sylwestrak E, O'Sullivan ML, Otto S, Tiglio K, Savas JN, Yates JR 3rd, Comoletti D, Taylor P, Ghosh A (2009) LRRTM2 interacts with Neurexin 1 and regulates excitatory synapse formation. Neuron 64:799-806.

DiAntonio A, Petersen SA, Heckmann M, Goodman CS (1999) Glutamate receptor expression regulates quantal size and quantal content at the Drosophila neuromuscular junction. J Neurosci 19:3023-3032.

Edery I, Zwiebel LJ, Dembinska ME, Rosbash M (1994) Temporal phosphorylation of the Drosophila period protein. Proc Natl Acad Sci U S A 91:2260-2264.

Featherstone DE, Rushton E, Rohrbough J, Liebl F, Karr J, Sheng Q, Rodesch CK, Broadie K (2005) An essential Drosophila glutamate receptor subunit that functions in both central neuropil and neuromuscular junction. J Neurosci 25:3199-3208.

Fire A, Xu S, Montgomery MK, Kostas SA, Driver SE, Mello CC (1998) Potent and specific genetic interference by double-stranded RNA in Caenorhabditis elegans. Nature 391:806-811.

Giagtzoglou N, Ly CV, Bellen HJ (2009) Cell adhesion, the backbone of the synapse: "vertebrate" and "invertebrate" perspectives. Cold Spring Harb Perspect Biol 1:a003079.

Gong WJ, Golic KG (2003) Ends-out, or replacement, gene targeting in Drosophila. Proc Natl Acad Sci U S A 100:2556-2561.

Graf ER, Zhang X, Jin SX, Linhoff MW, Craig AM (2004) Neurexins induce differentiation of GABA and glutamate postsynaptic specializations via neuroligins. Cell 119:1013-1026.

Guan B, Hartmann B, Kho YH, Gorczyca M, Budnik V (1996) The Drosophila tumor suppressor gene, $\mathrm{dlg}$, is involved in structural plasticity at a glutamatergic synapse. Curr Biol 6:695-706.

Harden N, Lee J, Loh HY, Ong YM, Tan I, Leung T, Manser E, Lim L (1996) A Drosophila homolog of the Rac- and Cdc42-activated serine/threonine kinase PAK is a potential focal adhesion and focal complex protein that colocalizes with dynamic actin structures. Mol Cell Biol 16:1896-1908.

Hoang B, Chiba A (2001) Single-cell analysis of Drosophila larval neuromuscular synapses. Dev Biol 229:55-70.

Ichtchenko K, Hata Y, Nguyen T, Ullrich B, Missler M, Moomaw C, Südhof TC (1995) Neuroligin 1: a splice site-specific ligand for beta-neurexins. Cell 81:435-443.

Ichtchenko K, Nguyen T, Südhof TC (1996) Structures, alternative splicing, and neurexin binding of multiple neuroligins. J Biol Chem 271:2676-2682.

Iida J, Hirabayashi S, Sato Y, Hata Y (2004) Synaptic scaffolding molecule is involved in the synaptic clustering of neuroligin. Mol Cell Neurosci 27:497-508.

Irie M, Hata Y, Takeuchi M, Ichtchenko K, Toyoda A, Hirao K, Takai Y, Rosahl TW, Südhof TC (1997) Binding of neuroligins to PSD-95. Science 277:1511-1515.

Jan LY, Jan YN (1976) Properties of the larval neuromuscular junction in Drosophila melanogaster. J Physiol 262:189-214.

Ko J, Fuccillo MV, Malenka RC, Südhof TC (2009a) LRRTM2 functions as a neurexin ligand in promoting excitatory synapse formation. Neuron 64:791-798.

Ko J, Zhang C, Arac D, Boucard AA, Brunger AT, Südhof TC (2009b) Neuroligin-1 performs neurexin-dependent and neurexin-independent functions in synapse validation. EMBO J 28:3244-3255.

Lahey T, Gorczyca M, Jia XX, Budnik V (1994) The Drosophila tumor suppressor gene dlg is required for normal synaptic bouton structure. Neuron 13:823-835.

Li C, Han D, Zhang F, Zhou C, Yu HM, Zhang GY (2007a) Preconditioning ischemia attenuates increased neurexin-neuroligin1-PSD-95 interaction after transient cerebral ischemia in rat hippocampus. Neurosci Lett 426:192-197.

Li J, Ashley J, Budnik V, Bhat MA (2007b) Crucial role of Drosophila neurexin in proper active zone apposition to postsynaptic densities, synaptic growth, and synaptic transmission. Neuron 55:741-755.

Margeta MA, Shen K (2010) Molecular mechanisms of synaptic specificity. Mol Cell Neurosci 43:261-267.

Marrus SB, DiAntonio A (2004) Preferential localization of glutamate receptors opposite sites of high presynaptic release. Curr Biol 14:924-931.

Marrus SB, Portman SL, Allen MJ, Moffat KG, DiAntonio A (2004) Differential localization of glutamate receptor subunits at the Drosophila neuromuscular junction. J Neurosci 24:1406-1415.

Meinertzhagen IA, Govind CK, Stewart BA, Carter JM, Atwood HL (1998) Regulated spacing of synapses and presynaptic active zones at larval neuromuscular junctions in different genotypes of the flies Drosophila and Sarcophaga. J Comp Neurol 393:482-492.

Meyer G, Varoqueaux F, Neeb A, Oschlies M, Brose N (2004) The complexity of PDZ domain-mediated interactions at glutamatergic synapses: a case study on neuroligin. Neuropharmacology 47:724-733.

Missler M, Zhang W, Rohlmann A, Kattenstroth G, Hammer RE, Gottmann K, Südhof TC (2003) Alpha-neurexins couple $\mathrm{Ca}^{2+}$ channels to synaptic vesicle exocytosis. Nature 423:939-948.

Nam CI, Chen L (2005) Postsynaptic assembly induced by neurexin-neuroligin interaction and neurotransmitter. Proc Natl Acad Sci U S A 102:6137-6142.

Packard M, Koo ES, Gorczyca M, Sharpe J, Cumberledge S, Budnik V (2002) The Drosophila Wnt, wingless, provides an essential signal for pre- and postsynaptic differentiation. Cell 111:319-330.

Petersen SA, Fetter RD, Noordermeer JN, Goodman CS, DiAntonio A (1997) Genetic analysis of glutamate receptors in Drosophila reveals a retrograde signal regulating presynaptic transmitter release. Neuron 19:1237-1248.

Qin G, Schwarz T, Kittel RJ, Schmid A, Rasse TM, Kappei D, Ponimaskin E, Heckmann M, Sigrist SJ (2005) Four different subunits are essential for expressing the synaptic glutamate receptor at neuromuscular junctions of Drosophila. J Neurosci 25:3209-3218.

Scheiffele P, Fan J, Choih J, Fetter R, Serafini T (2000) Neuroligin expressed in nonneuronal cells triggers presynaptic development in contacting axons. Cell 101:657-669.

Schmidt RL, Trejo TR, Plummer TB, Platt JL, Tang AH (2008) Infectioninduced proteolysis of PGRP-LC controls the IMD activation and melanization cascades in Drosophila. FASEB J 22:918-929.

Schuster CM, Ultsch A, Schloss P, Cox JA, Schmitt B, Betz H (1991) Molecular cloning of an invertebrate glutamate receptor subunit expressed in Drosophila muscle. Science 254:112-114.

Schuster CM, Davis GW, Fetter RD, Goodman CS (1996a) Genetic dissection of structural and functional components of synaptic plasticity. I. Fasciclin II controls synaptic stabilization and growth. Neuron 17:641-654.

Schuster CM, Davis GW, Fetter RD, Goodman CS (1996b) Genetic dissection of structural and functional components of synaptic plasticity. II. Fasciclin II controls presynaptic structural plasticity. Neuron 17:655-667.

Sigrist SJ, Thiel PR, Reiff DF, Schuster CM (2002) The postsynaptic glutamate receptor subunit DGluR-IIA mediates long-term plasticity in Drosophila. J Neurosci 22:7362-7372.

Spradling AC, Rubin GM (1982) Transposition of cloned P elements into Drosophila germ line chromosomes. Science 218:341-347.

Südhof TC (2008) Neuroligins and neurexins link synaptic function to cognitive disease. Nature 455:903-911.

Varoqueaux F, Aramuni G, Rawson RL, Mohrmann R, Missler M, Gottmann K, Zhang W, Südhof TC, Brose N (2006) Neuroligins determine synapse maturation and function. Neuron 51:741-754.

Vosshall LB, Wong AM, Axel R (2000) An olfactory sensory map in the fly brain. Cell 102:147-159.

Wan HI, DiAntonio A, Fetter RD, Bergstrom K, Strauss R, Goodman CS (2000) Highwire regulates synaptic growth in Drosophila. Neuron 26:313-329.

Wucherpfennig T, Wilsch-Bräuninger M, González-Gaitán M (2003) Role of Drosophila Rab5 during endosomal trafficking at the synapse and evoked neurotransmitter release. J Cell Biol 161:609-624.

Zeng X, Sun M, Liu L, Chen F, Wei L, Xie W (2007) Neurexin-1 is required for synapse formation and larvae associative learning in Drosophila. FEBS Lett 581:2509-2516. 